\title{
Effective Field Theory for jet substructure in heavy ion collisions
}

\author{
Varun Vaidya \\ Center for Theoretical Physics, Massachusetts Institute of Technology, \\ Cambridge, MA 02139, U.S.A. \\ E-mail: vvaidya@mit.edu
}

ABstRACT: I develop an Effective Field Theory (EFT) framework to compute jet substructure observables for heavy ion collision experiments. As an example, I consider dijet events that accompany the formation of a weakly coupled long lived Quark Gluon Plasma (QGP) medium in a heavy ion collision and look at an observable insensitive to jet selection bias: the simultaneous measurement of jet mass along with the transverse momentum imbalance between the jets that are groomed to remove soft radiation. Treating the jet as an open quantum system, I write down a factorization formula within the SCET (Soft Collinear Effective Theory) framework in the forward scattering regime. The physics of the medium is encoded in a universal soft field correlator while the jet-medium interaction is captured by a medium induced jet function. The factorization formula leads to a Lindblad type equation for the evolution of the reduced density matrix of the jet in the Markovian approximation. The solution for this equation allows a resummation of large logarithms that arise due to the final state measurements imposed while simultaneously summing over multiple incoherent interactions of the jet with the medium.

KeYwords: Perturbative QCD, Quark-Gluon Plasma, Resummation

ArXiv EPRINT: 2010.00028 


\section{Contents}

1 Introduction 1

2 The observable $\quad 4$

3 Factorization for reduced density matrix $r$

$\begin{array}{ll}3.1 \text { Leading order in Glauber: vacuum evolution } & 11\end{array}$

$\begin{array}{ll}3.2 \text { Next-to-leading order in Glauber } & 15\end{array}$

4 Decoherence of the hard interaction from medium scattering in a long lived medium

5 One loop results for medium induced functions

6 The master equation

7 Comparison with other formalisms

8 Summary and outlook

A Operator definitions and one loop results for vacuum evolution

A.1 Hard function

A.2 Jet function

A.3 Soft function

A.4 Resummation

\section{Introduction}

The natural final state of high energy hadronic or nuclear collisions are sprays of collimated particles consisting of hadrons and/or electrons. They are formed in an initial hard scattering, by which we mean a collision with a large transfer of momentum, followed by subsequent parton evolution know as a shower and fragmentation. Due to the large energies involved in jet production, they can be studied via perturbative QCD since QCD is weakly coupled at high virtualities of a parton. Therefore the calculation of the initial production of jets is under perturbative control, which makes jets powerful tools to probe the properties of the quark-gluon plasma (QGP) in heavy ion collisions.

This is based on the premise, which is now widely accepted that heavy ion collisions are the laboratory for the creation and study of the Quark Gluon Plasma medium. The high 
energy collision of nuclei both at RHIC and the LHC creates sufficiently energetic partons that can escape confinement from color neutral hadrons and give rise to a strongly/weakly coupled soup of quarks and gluons known as the Quark Gluon Plasma medium which, in thermal equilibrium is mainly characterized by its temperature. We can think of this plasma as consisting of soft partons with typical energy of the order of the temperature of the medium which is usually much lower than the center of mass energy of the initiating nuclear collision. The stopping collisions which create the QGP are accompanied by hard interactions which create highly energetic partons which eventually form jets. These jets then have to traverse through a region of the hot QGP as they evolve and hence they get modified in a heavy ion collision, compared with proton-proton collision, due to the jet-medium interaction.

We would therefore like to study the modification of jet substructure for the same hard event in Heavy Ion Collision (HIC) compared to a pp collision as a tool for extracting the properties of the medium. However, the selection of jets in a HIC suffers from the so called jet selection bias which is related to a phenomenon of jet quenching, that has been extensively studied in literature [1-20] and entails the systematic suppression of jet yields for a given radius (both small and large) and $p_{T}$ compared to pp collisions. This has been recently observed in experiments at both Relativistic Heavy Ion Collider (RHIC) and Large Hadron Collider (LHC) [21-30].The suppression mechanism happens through the mechanism of energy loss when jets travel through the hot medium. There has been tremendous theoretical effort to study the jet energy loss mechanism (see refs. [31-34] for recent reviews). This means that given a $p_{T}, R$ bin we are not comparing the evolution of the same hard events in pp versus HIC. One important goal of this paper is to propose observables that are insensitive to jet selection bias allowing us an apples to apples comparison for jet substructure.

The evolution of the jet in the medium usually depends on multiple scales such as the jet energy, the transverse momentum with respect to the jet axis, which will characterize the collinearity of the jet and thermal scales of the QGP. In current HIC experiments, the temperature achieved lies in the range $150-500 \mathrm{MeV}$, and may not always be a perturbative scale. Thus, a fully weak coupling calculation may not be valid. A hybrid model has been developed to address this problem [35-39], in which the initial jet production and vacuum-like parton shower are calculated perturbatively, while the subsequent jet energy loss in the medium is calculated by mapping the field theory computation in the strong coupling limit to a weak coupling computation in the classical gravity theory [40-45], i.e., by using a modification of the AdS/CFT correspondence [46].

However, since a holographic dual to QCD cannot be rigorously proven, it is unclear whether it can be used for precision jet substructure studies. In this paper, I will instead follow the idea that given a system with multiple hierarchically separated scales, a powerful tool is Effective Field Theory (EFT). The main advantage that an EFT approach offers compared to a purely perturbative Feynman diagram calculation is the notion of factorization, independent of perturbative order. This allows a separation of physics at widely separated scales in terms of manifestly gauge invariant operators which can be independently computed to any perturbative order desired. This approach has been applied with enormous success to study hadron structure, for e.g. in Deep Inelastic scattering (DIS) where factorization can 
be used to separate the perturbatively calculable hard function from the non-perturbative Parton Distribution Function (PDF). This establishes an operator definition for a universal hadron structure function which can then be either computed on the lattice or extracted from experiment.

Such an EFT approach in the context of HIC has been attempted previously in literature using a modification of Soft-Collinear Effective Theory [47-51], see [52] for review. The modification known as $\mathrm{SCET}_{G}$ uses the collinear sector of the corresponding EFT and this formalism has been used to address the question of jet quenching in the medium [53-57]. This approach treats the interaction of the jet with the medium in terms of a background gauge field, integrating out the propagating degrees of freedom of the medium. As a consequence, it is unclear how to establish manifest gauge invariance of operators or prove factorization rigorously in the presence of soft radiation.

I will use an alternative approach applying a new EFT for forward scattering that has been developed recently [58]. A key difference is that medium degrees of freedom are retained while integrating out the off-shell interaction between the jet and the medium to write manifestly gauge invariant interaction operators. I will show how this can be used to rigorously derive factorization formulas for jet substructure observables and will allow me to provide a operator definition for the QGP medium structure function in analogy with DIS.

The QGP medium exists for a very short time $(\sim 10 \mathrm{fm} / \mathrm{c})$ so that the jet spends a limited amount of time interacting with the medium. At the same time, the medium can be inhomogeneous and evolving with time so that the jet encounters a changing medium as it travels through it. Therefore, we need to keep track of the time evolution of the jet which is a novel feature compared to pp or DIS experiments. This can most easily be done by using the open quantum systems formalism (for introductory books, see $[59,60]$ ). For jets inside a QGP, if we only focus on jet observables, the jet can be treated as an open quantum system interacting with a QGP bath. The application of the open quantum system formalism in heavy ion collisions has been thriving in the study of color screening and regeneration of quarkonium [61-72]. The understanding of quarkonium in-medium dynamics has been improved by combining potential nonrelativistic QCD (pNRQCD [73-75], an EFT of QCD) and the open quantum system formalism [76-79]. For example, a semiclassical Boltzmann transport equation of quarkonium in the medium has been derived, under assumptions that are closely related with a hierarchy of scales [79-81].

In this paper, I will combine the tools of EFT with the open quantum system formalism and explore its physical implications for the jet-medium interaction. The long term goal is to develop a theoretically robust formalism for computing jet substructure observables for both light parton and heavy quark jets. For example, the bottom quark jets have been identified as an effective probe of the QGP medium and will be experimentally studied at LHCb, as well as by the sPHENIX collaboration at RHIC. There has been recent work on computing jet substructure observable for heavy quark jets in the context of proton-proton collisions $[82,83]$. The objective would then be to compute the same observables in heavy ion collisions and study modifications caused by the medium.

A first step was taken in [84] which looked at the transverse momentum spread of a single energetic quark as a function of the time of propagation through the QGP medium. 
However for a realistic description of the system, we also need to account for the initial hard interaction that creates the energetic quark which is dressed with radiation from the subsequent parton shower along with any medium interactions. At the same time a realistic final state jet produced in a heavy ion collision will usually have a large number of soft partons that originated from the QGP medium and are not directly associated with the evolution of the energetic jet. Thus any final state measurement imposed on the jet will have to account for these corrections which necessitates keeping track of the degrees of freedom of the QGP medium. A way around this, which has long been used to deal with soft contamination from Multi-Parton Interactions (MPIs) in pp collisions is that of jet grooming (for e.g. Soft-Drop [85]), which I ll use in this paper in simplified form.

To implement this, I will borrow the tools developed in the context of pp collision for computing groomed jet substructure observables. This field has progressed rapidly in recent years, both due to advances in explicit calculations, as well as due to the development of techniques for understanding properties of substructure observables using analytic [86] approaches. Developments in jet substructure (see [87] for a recent review) have shown that the modified mass drop tagging algorithm (mMDT) or soft-drop grooming procedure robustly removes contamination from both underlying event and non-global color-correlations, see ref. [85].

This paper is organized as follows: in section 2, I introduce the physical system that we wish to study along with the final state measurements imposed and the relevant physical scales that play an important role in its description. I also describe the relevant momentum modes that are dictated by these scales which will be a guide towards writing down a factorization formula. The next section 3 works out in detail, the factorization formula for the reduced density matrix of the jet within the framework of SCET. Section 4 deals with impact of the lack of coherence between the hard and medium interaction on the factorized density matrix due to the long-lived medium. In section 5 I present the one loop results for elastic collisions of the jet with the medium presenting its UV and IR structure. Section 6 gives the form of the master evolution equation for multiple jet-medium interactions and solves is analytically. Section 7 compares the formalism developed in this paper to previous approaches. Finally I conclude and discuss future directions in section 8. The details of the loop calculations for the vacuum and medium induced functions are given in appendix A and $\mathrm{B}$ respectively.

\section{The observable}

I want to a consider final state dijet event produced in a HIC in the background of a QGP medium. The jets are isolated using a suitable jet algorithm such as anti-kT with jet radius $R \sim 1$. We examine the scenario when the hard interaction creating the back to back jets happens at the periphery of the heavy ion collision, so that effectively only one jet passes through the medium while the other evolves purely in vacuum as shown in figure 1 . Since we do not want to keep track of the soft partons coming from the QGP, we groom the jets. We use the Soft-Drop algorithm [85] with $\beta=0$ and an energy cut-off sufficiently large to remove all partons at energy $\mathrm{T}$ and lower. Given a hard scale $\mathrm{Q} \sim 2 E_{J}$, where $E_{J}$ is the 


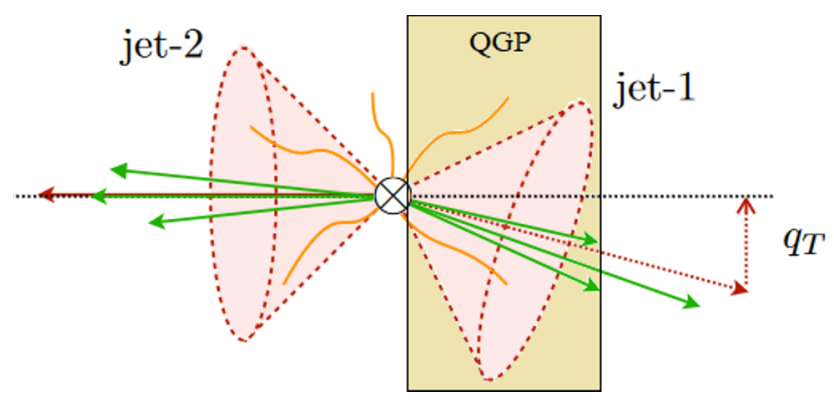

Figure 1. Dijet event in Heavy Ion collision at the periphery of the QGP medium.

energy of the jet and an energy cut-off, $z_{c} E_{J}$, we work in the hierarchy

$$
Q \sim z_{c} Q \gg T
$$

where $\mathrm{T}$ is the plasma temperature. The measurement we wish to impose is the transverse momentum imbalance between the two jets $q_{T} \sim T$. While this fixes the scaling of all radiation modes that fail grooming, this does not necessarily guarantee collinear scaling inside the jet. To ensure that, we also put in a cumulative jet mass, e, measurement on both jets with $Q \sqrt{e} \sim q_{T} \sim T$. An identical measurement with $z_{c} \ll 1$ for $e^{+} e^{-} \rightarrow$ dijets was discussed in [88] and we will refer the reader to that paper for a more detailed analysis of this observable.

We wish to write down a factorization theorem within Soft Collinear Effective Theory (SCET) which separates out functions depending on their scaling in momentum space. The philosophy is to divide up the phase space of possible radiative corrections according the scaling of the momentum modes which will capture the physics at leading power in $\lambda$. Once we identify the modes, like any other EFT, we write down an effective action obeying the necessary symmetry treating these modes as our degrees of freedom. Thus the idea is to implement factorization at the level of the action. This is to be contrasted with the approach of computing the observable in full QCD and then doing the expansion in $\lambda$ which is usually far more computationally challenging. At the same time, the EFT approach allows us to give operator definitions for factorized functions, many of which are universal (such as the PDF in pp collisions) which need not be recomputed for different observables. We have two scales in the problem, namely the hard scale Q and the IR scale T and we wish to capture the physics of two energetic back to back jets composed of light-like partons. Without loss of generality, we can assume the initiating $q \bar{q}$ pair created in the hard interaction to be moving along the $\mathrm{z}$ and $\mathrm{z}$ directions. This defines for us two reference light-like directions $n \equiv(1,0,0,1)$ and $\bar{n} \equiv(1,0,0,-1)$. For further analysis, we will work in light-cone co-ordinates, in which any momentum p can be decomposed as

$$
\begin{aligned}
p^{\mu} & =p^{-} \frac{n^{\mu}}{2}+p^{+} \frac{\bar{n}^{\mu}}{2}+p_{\perp}^{\mu}, \quad \text { or equivalently } \\
p^{\mu} & \equiv\left(p^{-}, p^{+}, \vec{p}_{\perp}\right)
\end{aligned}
$$

Given the scales and the reference light-like directions, we can carve up the momentum phase space in terms of different modes. First we have the so called "hard" mode which 
describes UV physics and consists of radiation which does not have a specific direction.

$$
p_{h}^{\mu} \sim Q(1,1,1), \text { Hard mode }
$$

Since the transverse momentum of this mode and hence its virtuality is of $\mathrm{O}(\mathrm{Q})$ (which $\gg$ our measurement $q_{T}$ ), no real radiation for this mode is possible but it can give virtual corrections. We integrate out this UV mode and its affect appears as the Wilson co-efficient of the operators in our effective action.

For our IR EFT, $\lambda=\frac{q_{T}}{Q} \sim \sqrt{e} \sim \frac{T}{Q} \ll 1$ is the expansion parameter and we will work to leading order in $\lambda$. The next obvious mode is the soft mode which again does not have a specific direction and scales uniformly in all its momentum components with the IR scale T. Along with any possible radiation that contributes to the measurement, this mode also captures the QGP degrees of freedom.

$$
p_{s}^{\mu} \sim Q(\lambda, \lambda, \lambda), \text { Global Soft mode }
$$

Finally, we have the collinear modes which describe the energetic degrees of freedom of the jet. The partons in the $n$ jet have "-" component of order Q, while its transverse momentum is fixed by the measurement to be $q_{T} \sim Q \sqrt{e_{n}} \sim \lambda Q$. The scaling of the "+" component is then fixed by demanding $p^{+} p^{-} \sim p_{\perp}^{2}$, which follows from the observation that the radiative corrections at leading power in $\lambda$ are captured by radiation collinear (as well as soft radiation already considered) to the initiating $q \bar{q}$ pair and with virtuality $Q \lambda$.

$$
p_{n}^{\mu} \sim Q\left(1, \lambda^{2}, \lambda\right), \text { collinear mode }
$$

Similarly, we can fix the scaling of the $\bar{n}$ collinear mode,

$$
p_{\bar{n}}^{\mu} \sim Q\left(\lambda^{2}, 1, \lambda\right), \text { collinear mode }
$$

In principle, we can have another mode which is not a propagating degree of freedom but mediates an instantaneous coulomb-like interaction between the Soft and collinear modes. This is known as the Glauber mode. The scaling of this mode is fixed by demanding that a Glauber exchange should maintain the momentum scaling of the Soft and collinear modes and thus enforce forward scattering. We can have three types of Glauber modes mediating interactions between $n-\bar{n}, \bar{n}-s$ and $n-s$ modes

$$
\begin{aligned}
p_{G, n \bar{n}}^{\mu} & \sim Q\left(\lambda^{2}, \lambda^{2}, \lambda\right) \\
p_{G, \bar{n} s}^{\mu} & \sim Q\left(\lambda^{2}, \lambda, \lambda\right) \\
p_{G, n s}^{\mu} & \sim Q\left(\lambda, \lambda^{2}, \lambda\right)
\end{aligned}
$$

For most jet observables in vacuum, and, in particular the one which we are considering, these modes are redundant in that their effect is already captured by the Soft and collinear physics. We refer the reader to [58] for more details. So for pure vacuum evolution such an in e-p, $e^{+} e^{-}$or $p p$ these modes are irrelevant. However, in our case we also have a medium which is made up of Soft modes, and an $n$ jet which can interact with it. Indeed, 
the interaction of a jet in the medium is dominated by forward scattering so that we need to include the $p_{G, n s}$ Glauber mode in our EFT. In the next section, we will look at the structure of the effective action in terms of all of relevant modes described thus far.

The medium induces another scale, namely the Debye screening mass $m_{D} \sim g T$. We will work in a weakly coupled regime so that $m_{D}$ is a much smaller scale than T. In a completely quantum coherent process such as a pp collision, the imposition of an IRC (Infra-Red collinear) safe observable on the final state guarantees that the final state physics is not sensitive to any IR scale below the one set by the measurement. However, in our case the presence of the medium can induce incoherent scattering which can potentially make the observable sensitive to $m_{D}$ as we will see in an explicit calculation.

Given the scaling we see that the soft mode fails grooming. The emissions that fail grooming lie outside the groomed jet and hence contribute to the transverse momentum imbalance. The collinear mode is sensitive to the grooming parameter $z_{c}$ so that it may either pass or fail grooming. When it fails grooming, it will contribute to the transverse momentum imbalance while when it passes, it will contribute to the cumulative jet mass. We assume that the particles that make up the medium also scale uniformly in temperature and have the same scaling as the soft mode.

From the scaling of the modes, we see that the constraints from the observable imposed imply that there is no radiation that is sensitive to the edge of the jet and hence to the jet radius. The soft modes all fail grooming and lie outside the groomed jet while the collinear radiation is confined to a narrow core near the jet axis. Hence, there are no sources of energy/ transverse momentum leakage near the edge of the jet. There will be jet substructure modification within the jet without changing its radius and $p_{T}$ so that it now becomes possible to compare the jet substructure modification for the same hard events in pp and HIC even while working in the same $p_{T}, \mathrm{R}$ bin.

\section{Factorization for reduced density matrix}

I would like to derive a factorization formula for this observable showing a clear separation of scales in terms of gauge invariant operators. I will treat the jet as an open quantum system interacting with the QGP bath and follow the time evolution of the reduced density matrix of the jet. Along the way, I will work out the factorization for this density matrix within the framework of SCET.

For ease of analysis, we consider that the hard interaction that creates the jet is an $e^{+} e^{-}$ collision. While this is not a real scenario, it is an ideal playground to work out the EFT framework which mainly deals with the final state physics. The EFT structure can then be easily carried over to the realistic case of nuclear/hadronic collisions and I will outline how this can be done in section 8 . While a sketch of the factorization for the same observable with a different hierarchy of scales was provided in [88], we revisit the factorization in the context of a density matrix evolution, which uses time ordered perturbation theory.

The hard interaction can be encoded using an effective current operator

$$
\mathcal{O}_{H}=C(Q) L_{\mu} J_{\text {SCET }}^{\mu}
$$


$C(Q)$ is the Wilson co-efficient for this contact operator that depends only on the hard scale Q. This will lead to a hard function $\mathrm{H}(\mathrm{Q})$ at the amplitude squared level and its form is discussed in appendix A. $L_{\mu}$ is the initial state current, which in this case is just the lepton current, while $J_{\text {SCET }}^{\mu}$ is the final state SCET current, which is just the gauge invariant quark current.

$$
L^{\mu}=\bar{l} \gamma^{\mu} l
$$

The SCET current is given as

$$
J_{\mathrm{SCET}}^{\mu}=\left[S_{n}^{\dagger} S_{\bar{n}}\right] \bar{\chi}_{n} W_{n}^{\dagger} \gamma^{\mu} W_{\bar{n}} \chi_{\bar{n}}
$$

where $S_{i}, W_{j}$ are soft and collinear Wilson lines, defined as

$$
\begin{aligned}
S_{n}^{(r)}(x) & =\mathrm{P} \exp \left[i g \int_{-\infty}^{0} d s n \cdot A_{s}^{B}(x+s n) T_{(r)}^{B}\right] \\
W_{n}(x) & =\left[\sum_{\text {perms }} \exp \left(-\frac{g}{n \cdot \mathcal{P}} \bar{n} \cdot A_{n}(x)\right)\right]
\end{aligned}
$$

$\chi_{n}$ is the $\mathrm{n}$ collinear quark field expanded out to leading order in $\lambda . \mathcal{P}^{\mu}$ acts like the derivative operator and acts on the field to its right (in this case $A_{n}(x)$ ) to extract out the momentum of the states created/annihilated by the field. We see that the SCET current is already factorized in terms of soft and collinear sectors which are manifestly gauge invariant due to the presence of Wilson lines. This form of the SCET current is correct to leading order in our power counting parameter $\lambda$. Operationally, the collinear Wilson line encodes the n collinear emissions from the $\bar{n}$ collinear sector to leading power while the $S_{n}$ and $S_{\bar{n}}$ soft wilson lines encode the soft emissions from the $\mathrm{n}$ and $\bar{n}$ collinear sectors, again to leading power in $\lambda$. Thus the interaction between the $\mathrm{n}, \bar{n}$ and soft sectors is now completely captured in these Wilson lines so that these sectors are now decoupled at the level of the action, i.e., the effective Hamiltonian can be written as

$$
H^{\mathrm{SCET}}=H_{n}+H_{\bar{n}}+H_{s}+O\left(\lambda^{2}\right)
$$

However, as we shall see, there is also a Glauber induced interaction between the sectors which cannot be decoupled in the form of Wilson lines since it does not eikonalize and needs to be added explicitly to the Hamiltonian. The initial state density matrix is given as

$$
\rho(0)=\left|e^{+} e^{-}\right\rangle\left\langle e^{+} e^{-}\right| \otimes \rho_{B}
$$

$\rho_{B}$ is the density matrix of the medium, which we assume to be time independent in this paper. This is not strictly necessary for proving factorization and a time dependence can be introduced, which would be equivalent to an inhomogeneity in the medium from the perspective of the jet. I will comment on this after eq. (3.46) where we use the translational invariance of the QGP medium. Since the partons in the medium have the same scaling as the soft mode, we will henceforth suppress explicitly writing out the factor $\rho_{B}$ till it becomes relevant for the soft function analysis. We have started with the assumption that 
the initial state participating in the hard interaction is disentangled from the state of the background medium. We can follow the evolution of this density matrix which will evolve with the effective Hamiltonian

$$
H=H^{\mathrm{IR}}+\mathcal{O}_{H}
$$

The IR Hamiltonian is written as a sum over the Hamiltonians of all the SCET sectors (i.e., the $\mathrm{n}$ collinear, $\bar{n}$ collinear, soft) along with the Glauber Hamiltonian, which in our case introduces a interaction between the soft and $\mathrm{n}$ collinear sectors.

$$
H^{\mathrm{IR}}=H^{\mathrm{SCET}}+H^{G}=H_{n}+H_{\bar{n}}+H_{s}+H_{n s}^{G}
$$

This form of the QCD Hamiltonian is correct to leading power in our expansion parameter $\lambda$. We see here that the three sectors $\mathrm{n}, \bar{n}$ and soft are decoupled in the $H^{\mathrm{SCET}}$ Hamiltonian and only talk to each other via the hard $O_{H}$ and Glauber $H_{n s}^{G}$ terms. The interaction induced by the Glauber mode does not eikonalize in the form of Wilson lines and hence it explicitly induces a coupling between the $\mathrm{n}$ collinear and soft sectors at the level of the action. Only the $H_{n s}^{G}$ Galuber Hamiltonian that couples the $\mathrm{n}$ jet and the medium soft degrees of freedom is included since only the $\mathrm{n}$ jet interacts with the medium. We will not describe the details for the Hamiltonians of the individual sectors $H_{n}, H_{\bar{n}}$ and $H_{S}$, which are standard in SCET literature but refer the reader to a review of SCET [52]. The Hamiltonian of each of the 3 sectors can be further written as a sum over the free theory Hamiltonian and an interaction term. For instance

$$
H_{n}=H_{n}^{0}+H_{n, \text { int }}
$$

where $H_{n \text {,int }}$ describes the interaction between the $\mathrm{n}$ collinear degrees of freedom. As motivated in [84], the dominant interaction of the jet with the medium is mediated by the $t$ channel exchange of the Glauber gluon. The Glauber Hamiltonian is expressed in terms of effective gauge invariant operators for quark-quark $(q q)$, quark-gluon ( $q g$ or $g q)$ and gluon-gluon $(g g)$ interactions which have been worked out in the Feynman gauge in ref. [58]

$$
\begin{aligned}
& H_{n s}^{G}=\sum_{i j} C_{i j} \mathcal{O}_{n s}^{i j} \\
& \mathcal{O}_{n s}^{q q}=\mathcal{O}_{n}^{q B} \frac{1}{\mathcal{P}_{\perp}^{2}} \mathcal{O}_{s}^{q_{n} B}, \quad \mathcal{O}_{n s}^{q g}=\mathcal{O}_{n}^{q B} \frac{1}{\mathcal{P}_{\perp}^{2}} \mathcal{O}_{s}^{g_{n} B}, \\
& \mathcal{O}_{n s}^{g q}=\mathcal{O}_{n}^{g B} \frac{1}{\mathcal{P}_{\perp}^{2}} \mathcal{O}_{s}^{q_{n} B}, \quad \mathcal{O}_{n s}^{g g}=\mathcal{O}_{n}^{g B} \frac{1}{\mathcal{P}_{\perp}^{2}} \mathcal{O}_{s}^{g_{n} B}
\end{aligned}
$$

where $B$ is the color index and the subscripts $n$ and $s$ denote the collinear and soft operators. The Glauber gluon propagator appears as the derivative in $\perp$ direction. We will assume for the remainder of the paper that the jet which traverses the medium points along the $\mathrm{n}$ direction. $C_{i j}$ are the Wilson co-efficients for these contact operators and all begin at $O\left(\alpha_{s}\right)$. Since I am interested in a dijet event, I will consider only a single insertion of the hard operator which, at tree level will create a back to back $q \bar{q}$ pair that subsequently evolves into dijets. The time evolved density matrix is given as

$$
\begin{aligned}
\rho(t) & =e^{-i H t} \rho(0) e^{i H t}=e^{-i H^{\mathrm{IR}} t}\left[e^{i H^{\mathrm{IR}} t} e^{-i H t}\right] \rho(0)\left[e^{i H t} e^{-i H^{\mathrm{IR}} t}\right] e^{i H^{\mathrm{IR}} t} \\
& =e^{-i H^{\mathrm{IR}}} U(t, 0) \rho(0) U^{\dagger}(t, 0) e^{i H^{\mathrm{IR}} t}
\end{aligned}
$$


Our evolution operator $U(t, 0)$ now obeys the equation

$$
\partial_{t} U(t, 0)=-i \mathcal{O}_{H, \mathrm{IR}}(t) U(t, 0), \quad \text { with } \quad \mathcal{O}_{H, \mathrm{IR}}(t)=e^{i H^{\mathrm{IR}} t} \mathcal{O}_{H} e^{-i H^{\mathrm{IR}} t}
$$

which has the solution

$$
U(t, 0)=\mathcal{T}\left\{e^{-i \int_{0}^{t} d t^{\prime} \mathcal{O}_{H, \mathrm{IR}}\left(t^{\prime}\right)}\right\}
$$

which is the evolution operator written as a time ordered exponent of the hard operator dressed with the IR Hamiltonian. Our solution for the density matrix now becomes

$$
\rho(t)=e^{-i H^{\mathrm{IR}}} t \mathcal{T}\left\{e^{-i \int_{0}^{t} d t^{\prime} \mathcal{O}_{H, \mathrm{IR}}\left(t^{\prime}\right)}\right\} \rho(0) \overline{\mathcal{T}}\left\{e^{-i \int_{0}^{t} d t^{\prime} \mathcal{O}_{H, \mathrm{IR}}\left(t^{\prime}\right)}\right\} e^{i H^{\mathrm{IR}} t}
$$

We are interested in creating dijets, it is sufficient to consider a single insertion of the Hard operator on each side of the cut.

$$
\rho(t)=e^{-i H^{\mathrm{IR}} t} \rho(0) e^{i H^{\mathrm{IR}} t}+\int_{0}^{t} d t_{1} \int_{0}^{t} d t_{2} e^{-i H^{\mathrm{IR}} t} \mathcal{O}_{H, \mathrm{IR}}\left(t_{1}\right) \rho(0) \mathcal{O}_{H, \mathrm{IR}}^{\dagger}\left(t_{2}\right) e^{i H^{\mathrm{IR}} t}
$$

When we impose the measurement for the dijet event with the required properties, only the second terms will survive, hence hereafter we can simply follow the evolution for this piece.

We define

$$
\begin{aligned}
\sigma(t)= & \int_{0}^{t} d t_{1} \int_{0}^{t} d t_{2} e^{-i H^{\mathrm{IR}}} \mathcal{O}_{H, \mathrm{IR}}\left(t_{1}\right) \rho(0) \mathcal{O}_{H, \mathrm{IR}}^{\dagger}\left(t_{2}\right) e^{i H^{\mathrm{IR}} t} \\
= & |C(Q)|^{2} I_{\mu \nu} \int d^{3} x_{1} \int_{0}^{t} d t_{1} \int d^{3} x_{2} \int_{0}^{t} d t_{2} e^{-i\left(x_{1}-x_{2}\right) \cdot\left(p_{e}+p_{\bar{e}}\right)} \\
& e^{-i H^{\mathrm{IR}} t} J_{\mathrm{SCET}, \mathrm{IR}}^{\mu}\left(x_{1}\right)|0\rangle\langle 0| J_{\mathrm{SCET}, \mathrm{IR}}^{\nu}\left(x_{2}\right) e^{i H^{\mathrm{IR}} t}
\end{aligned}
$$

where $p_{e}, p_{\bar{e}}$ are the momenta of the initial state electron positron. In the c.o.m. frame $p_{e}+p_{\bar{e}}=(Q, 0,0,0) . I^{\mu \nu}$ is the Lepton tensor.

However, the Glauber Hamiltonian prevents us from factorizing the full density matrix since it couples the collinear $\mathrm{n}$ and the Soft sectors. We therefore need to expand in powers of the Glauber Hamiltonian and establish factorization at each order in the Glauber expansion. We will show that after imposing final state measurements, it is possible to resum the series in the Glauber Hamiltonian, atleast in the Markovian approximation via a Lindblad type equation.

To proceed, we rearrange the result above so as to be able to do a systematic expansion in the Glauber Hamiltonian. For convenience lets define

$$
\begin{aligned}
\int d \tilde{x}= & \int d^{3} x_{1} \int_{0}^{t} d t_{1} \int d^{3} x_{2} \int_{0}^{t} d t_{2} e^{-i\left(x_{1}-x_{2}\right) \cdot\left(p_{e}+p_{\bar{e}}\right)} \\
\sigma(t)= & |C(Q)|^{2} I_{\mu \nu} \int d \tilde{x} e^{-i H^{\mathrm{IR}} t} J_{\mathrm{SCET}, \mathrm{IR}}^{\mu}\left(x_{1}\right)|0\rangle\langle 0| J_{\mathrm{SCET}, \mathrm{IR}}^{\nu}\left(x_{2}\right) e^{i H^{\mathrm{IR}} t} \\
= & |C(Q)|^{2} I^{\mu \nu} \int d \tilde{x} e^{-i H^{\mathrm{SCET}}} t\left\{e^{i H^{\mathrm{SCET}}} t e^{-i H^{\mathrm{IR}}\left(t-t_{1}\right)} e^{-i H^{\mathrm{SCET}} t_{1}}\right\} \\
& \left\{e^{i H^{\mathrm{SCET}}} t_{1} J_{\mathrm{SCET}}^{\mu}\left(\vec{x}_{1}, 0\right) e^{-i H^{\mathrm{SCET}} t_{1}}\right\}\left\{e^{i H^{\mathrm{SCET}} t_{1}} e^{-i H^{\mathrm{IR}} t_{1}}\right\}|0\rangle\langle 0|\left\{e^{i H^{\mathrm{IR}} t_{2}} e^{-i H^{\mathrm{SCET}} t_{2}}\right\} \\
& \left\{e^{i H^{\mathrm{SCET}}} t_{2} J_{\mathrm{SCET}}^{\nu}\left(\vec{x}_{2}, 0\right) e^{-i H^{\mathrm{SCET}} t_{2}}\right\}\left\{e^{i H^{\mathrm{SCET}} t_{2}} e^{i H^{\mathrm{IR}}\left(t-t_{2}\right)} e^{-i H^{\mathrm{SCET}} t}\right\} e^{i H^{\mathrm{SCET}} t}
\end{aligned}
$$


Using the same process as for the hard operator, we can rearrange the expression as time ordered dressed operators

$$
\begin{aligned}
& \sigma(t)=|C(Q)|^{2} I_{\mu \nu} \int d \tilde{x} e^{-i H^{\mathrm{SCET}} t} \mathcal{T}\left\{e^{-i \int_{0}^{t} d t^{\prime} H_{I_{s c}}^{G}\left(t^{\prime}\right)} J_{\mathrm{SCET}, I_{s c}}^{\mu}\left(x_{1}\right)\right\}|0\rangle \\
& \langle 0| \overline{\mathcal{T}}\left\{e^{-i \int_{0}^{t} d t^{\prime} H_{I_{s c}}^{G}\left(t^{\prime}\right)} J_{\mathrm{SCET}, I_{s c}}^{\nu}\left(x_{2}\right)\right\} e^{i H^{\mathrm{SCET}} t}
\end{aligned}
$$

where

$$
O_{I_{s c}}(t)=e^{i H^{\mathrm{SCET}} t} O e^{-i H^{\mathrm{SCET}} t}
$$

so that all operators are now dressed with the SCET Hamiltonian. We are now set up to do an expansion in the Glauber Hamiltonian. Ultimately, for this paper, we want to compute the trace over the reduced density matrix with an appropriate measurement

$$
\left.\Sigma(t) \equiv \operatorname{Tr}[\sigma(t) \mathcal{M}]\right|_{t \rightarrow \infty}
$$

where we first completely trace over the Soft and collinear degrees of freedom with the measurement $\mathcal{M}$ which includes the jet algorithm to isolate a final state large radius groomed dijet configuration with the required $q_{T}$ imbalance and jet mass. We then expand this out in powers of $H^{G}$

$$
\Sigma(t)=\Sigma^{(0)}(t)+\Sigma^{(1)}(t)+\Sigma^{(2)}(t)+O\left(\left[H^{G}\right]^{3}\right)+\ldots
$$

In the next section we will sketch the proof for factorization of the reduced density matrix upto quadratic order in the $H^{G}$ expansion. We will subsequently use this to derive a Lindblad equation to resum all the higher order terms in $H^{G}$ in the Markovian approximation.

\subsection{Leading order in Glauber: vacuum evolution}

We start with the leading order term from eq. (3.18) with no Glauber insertions and so should simply give us a result proportional to the vacuum-background cross section. Since we are doing this in the context of time ordered perturbation theory we will outline the proof for factorization of this piece here.

$$
\begin{aligned}
\sigma^{(0)}(t)= & |C(Q)|^{2} I_{\mu \nu} \int d \tilde{x} e^{-i H^{\mathrm{SCET}}\left(t-t_{1}\right)} J_{\mathrm{SCET}}^{\mu}\left(\vec{x}_{1}\right) e^{-i H^{\mathrm{SCET}} t_{1}}|0\rangle \\
& \langle 0| e^{i H^{\mathrm{SCET}} t_{2}} J_{\mathrm{SCET}}^{\nu}\left(\vec{x}_{2}\right) e^{i H^{\mathrm{SCET}}\left(t-t_{2}\right)}
\end{aligned}
$$

We can now write the result in the interaction picture as $H_{\text {int }}$,

$$
H^{\mathrm{SCET}}=H^{0}+H_{\mathrm{int}}
$$

where $H^{0}, H_{\text {int }}$ respectively is a sum over the free and interaction Hamiltonians for the $\mathrm{n}$, $\bar{n}$ and Soft sectors. Then performing the same series of steps as before, we can write our result in terms of the free theory interaction picture,

$$
\begin{aligned}
\sigma^{(0)}(t)= & |C(Q)|^{2} I_{\mu \nu} \int d \tilde{x} e^{-i H^{0} t} \mathcal{T}\left\{e^{-i \int_{0}^{t} d t^{\prime} H_{\mathrm{int}, I}\left(t^{\prime}\right)} J_{\mathrm{SCET}, I}^{\mu}\left(x_{1}\right)\right\}|0\rangle \\
& \langle 0| \overline{\mathcal{T}}\left\{e^{-i \int_{0}^{t} d t^{\prime} H_{\mathrm{int}, I}\left(t^{\prime}\right)} J_{\mathrm{SCET}, I}^{\nu}\left(x_{2}\right)\right\} e^{i H^{0} t}
\end{aligned}
$$


where

$$
O_{I}(t)=e^{i H^{0} t} O e^{-i H^{0} t}
$$

so that all the operators are now dressed with the free theory Hamiltonian. To proceed further, we put in our measurement on the dijets and take a trace over final states and take the limit $t \rightarrow \infty$.

$$
\begin{aligned}
& \langle X|\sigma(t \rightarrow \infty) \mathcal{M}| X\rangle \equiv \Sigma^{(0)}=|C(Q)|^{2} I_{\mu \nu} \int d \tilde{x} \\
& \left\langle X\left|\mathcal{T}\left\{e^{-i \int_{0}^{\infty} d t^{\prime} H_{\text {int }, I}\left(t^{\prime}\right)} J_{\text {SCET }, I}^{\mu}\left(x_{1}\right)\right\}\right| 0\right\rangle\left\langle 0\left|\overline{\mathcal{T}}\left\{e^{-i \int_{0}^{\infty} d t^{\prime} H_{\text {int }, I}\left(t^{\prime}\right)} J_{\text {SCET }, I}^{\nu}\left(x_{2}\right)\right\} \mathcal{M}\right| X\right\rangle
\end{aligned}
$$

where we have

$$
\mathcal{M}=\delta^{2}\left(\vec{q}_{T}-\vec{p}_{X_{n}, \in \mathrm{gr}}^{\perp}-\vec{p}_{X_{\bar{n}}, \in \mathrm{gr}}^{\perp}\right) \Theta\left(e_{n}-e_{n, \in \mathrm{gr}}\right) \Theta\left(e_{\bar{n}}-e_{\bar{n}, \in \mathrm{gr}}\right)
$$

where $\vec{q}_{T}$ is the transverse momentum imbalance between the two groomed jets, while $e_{n}$, $e_{\bar{n}}$ measures the jet mass for the two groomed jets. The jet mass is defined as

$$
e_{n, \in \mathrm{gr}}=\frac{4}{Q^{2}}\left(\sum_{i \in n, \mathrm{gr}} p_{X_{i}}\right)^{2}
$$

Notice that none of the modes are sensitive to the jet radius $\mathrm{R}$. This follows from the fact that the collinear radiation is collimated along the jet axis far away from the edge of the jet while the soft radiation can populate the full phase space without constraint. Our Hamiltonian is

$$
H_{\text {int }}=H_{s, \text { int }}+H_{n, \text { int }}+H_{\bar{n}, \text { int }}
$$

where the three sectors are decoupled from each other while the interactions between the various sectors now appear in the form of Wilson lines in the SCET current (eq. (3.3)). Without the presence of factorization violating Glauber interactions, using standard techniques outlined in [88], the momentum sectors can now be written as separate matrix elements. Hence the Hilbert space also factorizes into various momentum mode states

$$
|X\rangle=\left|X_{n}\right\rangle\left|X_{\bar{n}}\right\rangle\left|X_{s}\right\rangle
$$

Since the Hamiltonian is already factorized, we have, in principle, a factorization of all the modes at this stage. However, we still have to implement our power counting on the measurement functions which will ensure that only leading power corrections in our expansion parameter $\lambda$ are retained.

Acting on the final state Hilbert space, we can then pull out the co-ordinate dependence of the SCET current and perform all co-ordinate integrals

$$
\begin{aligned}
\Sigma^{(0)}= & |C(Q)|^{2} I_{\mu \nu} \int d^{4} x_{1} \int d^{4} x_{2} e^{-i\left(x_{1}-x_{2}\right) \cdot\left(p_{e}+p_{\bar{e}}-p_{X_{n}}-p_{X_{\bar{n}}}-p_{X_{s}}\right)} \\
& \times\left\langle X\left|\mathcal{T}\left\{e^{-i \int_{0}^{\infty} d t^{\prime} H_{\mathrm{int}, I}\left(t^{\prime}\right)} J_{\mathrm{SCET}, I}^{\mu}(0)\right\}\right| 0\right\rangle\left\langle 0\left|\overline{\mathcal{T}}\left\{e^{-i \int_{0}^{\infty} d t^{\prime} H_{\mathrm{int}, I}\left(t^{\prime}\right)} J_{\mathrm{SCET}, I}^{\nu}(0)\right\} \mathcal{M}\right| X\right\rangle
\end{aligned}
$$


Performing the integrals over $x_{1}$ and $x_{2}$ now gives momentum conserving $\delta$ function along with a $4 \mathrm{~d}$ volume factor $\mathrm{V}$. We can then decompose the 4 momentum delta function in light-cone co-ordinates and apply power counting

$$
\begin{aligned}
& \delta^{4}\left(p_{e}+p_{\bar{e}}-p_{X_{n}}-p_{X_{\bar{n}}}-p_{X_{s}}\right) \\
\rightarrow & \delta\left(Q-p_{X_{n}}^{-}\right) \delta\left(Q-p_{X_{\bar{n}}}^{+}\right) \delta^{2}\left(p_{X_{n, \in \mathrm{gr}}}^{\perp}+p_{\bar{X}_{\bar{n}, \in \mathrm{gr}}}^{\perp}+p_{X_{s}}^{\perp}+p_{X_{n, \notin \mathrm{gr}}}^{\perp}+p_{\bar{X}_{\bar{n}, \notin \mathrm{gr}}}^{\perp}\right)
\end{aligned}
$$

An example of how power counting works in this expression is that for the momentum conservation in the "-" light-cone direction, the contribution of the n sector scales as Q, while the contributions from the $\bar{n}$ and soft sectors which respectively scale as $\lambda^{2} Q$ and $\lambda Q$ are power suppressed and hence we drop them. The same is true for the "+" component where only the $\bar{n}$ sector is relevant. Finally for the $\perp$ direction, all momenta scale uniformly as $\lambda Q$ and hence all contributions are relevant. We will apply this logic again while deriving factorization at higher orders in the Glauber expansion. The subscript $\notin$ gr indicates collinear radiation that fails the grooming condition. Our factorization now becomes

$$
\begin{aligned}
\Sigma^{(0)}= & V \times|C(Q)|^{2} I_{\mu \nu}\left\langle X\left|\mathcal{T}\left\{e^{-i \int_{0}^{\infty} d t^{\prime} H_{\mathrm{int}, I}\left(t^{\prime}\right)} J_{\mathrm{SCET}, I}^{\mu}(0)\right\}\right| 0\right\rangle \\
& \left\langle 0\left|\overline{\mathcal{T}}\left\{e^{-i \int_{0}^{\infty} d t^{\prime} H_{\mathrm{int}, I}\left(t^{\prime}\right)} J_{\mathrm{SCET}, I}^{\nu}(0)\right\}\right| X\right\rangle \\
& \times \delta\left(Q-p_{X_{n}}^{-}\right) \delta\left(Q-p_{X_{\bar{n}}}^{+}\right) \delta^{2}\left(p_{X_{n, \in \mathrm{gr}}}^{\perp}+p_{\bar{X}_{\bar{n}, \in \mathrm{gr}}}^{\perp}+p_{X_{s}}^{\perp}+p_{X_{n, \notin \mathrm{gr}}}^{\perp}+p_{\bar{X}_{\bar{n}, \notin \mathrm{gr}}}^{\perp}\right) \\
& \times \delta^{2}\left(\vec{q}_{T}-\vec{p}_{\bar{X}_{n}, \in \mathrm{gr}}^{\perp}-\vec{p}_{\bar{X}_{\bar{n}}, \in \mathrm{gr}}^{\perp}\right) \Theta\left(e_{n}-e_{n, \in \mathrm{gr}}\right) \Theta\left(e_{\bar{n}}-e_{\bar{n}, \in \mathrm{gr}}\right)
\end{aligned}
$$

Without loss of generality, we can assume that the axis of the $\bar{n}$ ungroomed jet is exactly aligned with the $\bar{n}$ direction, in which case its transverse momentum becomes zero.

$$
\begin{aligned}
\Sigma^{(0)}= & V \times|C(Q)|^{2} I_{\mu \nu}\left\langle X\left|\mathcal{T}\left\{e^{-i \int_{0}^{\infty} d t^{\prime} H_{\mathrm{int}, I}\left(t^{\prime}\right)} J_{\mathrm{SCET}, I}^{\mu}(0)\right\}\right| 0\right\rangle \\
& \left\langle 0\left|\overline{\mathcal{T}}\left\{e^{-i \int_{0}^{\infty} d t^{\prime} H_{\mathrm{int}, I}\left(t^{\prime}\right)} J_{\mathrm{SCET}, I}^{\nu}(0)\right\} \mathcal{M}\right| X\right\rangle \\
& \times \delta\left(Q-p_{X_{n}}^{-}\right) \delta\left(Q-p_{X_{\bar{n}}}^{+}\right) \delta^{2}\left(\vec{q}_{T}+p_{X_{s}}^{\perp}+p_{X_{n, \notin \mathrm{gr}}}^{\perp}+p_{\bar{X}_{\bar{n}, \notin \mathrm{gr}}}^{\perp}\right) \delta_{\mathcal{P}_{\bar{n}}^{\perp}}^{2} \\
& \times \delta^{2}\left(p_{X_{s}}^{\perp}-\vec{p}_{\bar{X}_{n}}^{\perp}\right) \Theta\left(e_{n}-e_{n, \in \mathrm{gr}}\right) \Theta\left(e_{\bar{n}}-e_{\bar{n}, \in \mathrm{gr}}\right)
\end{aligned}
$$

$\delta_{\mathcal{P}_{\bar{n}}^{\perp}}$ is a Kronecker delta setting the transverse momentum of the $\bar{n}$ ungroomed jet to 0 . We now have a transverse momentum condition $\delta^{2}\left(p_{X_{s}}^{\perp}-\vec{p}_{X_{n}}^{\perp}\right)$, which tells us that the ungroomed $n$ jet is not exactly aligned with the $\mathrm{n}$ axis. However, we can use type 1 Reparameterization invariance (RPI 1) symmetry of SCET [89], to adjust the axis of the jet without changing any physics. When we choose specific light-like directions $\mathrm{n}$ and $\bar{n}$, we explicitly break Lorentz symmetry of the QCD Lagrangian. However, there is a residual Lorentz symmetry left over that allows us to make the transformation $n_{\mu} \rightarrow n_{\mu}+\Delta_{\mu}^{\perp}$ so long as the shift by $\Delta^{\perp}$ does not change the momentum scaling of the collinear mode. So this allows a shift of up to $Q \lambda \sim p_{X_{s}}^{\perp}$ in the perpendicular component of momentum for the whole collinear sector. This allows us to write $\delta^{2}\left(\vec{p}_{X_{n}}^{\perp}\right)$ which now gets us back to the standard definition of the jet function. We also see that the transverse momentum imbalance $\vec{q}_{T}$ receives contributions from all the modes that fail grooming which is what we expect. 
We can convert the Kronecker delta to a Dirac delta following literature [90]

$$
\delta_{\mathcal{P}_{\bar{n}}^{\perp}}^{2}=\pi Q^{2} \delta^{2}\left(p_{\bar{X}_{\bar{n}}, \in \mathrm{gr}}^{\perp}\right)
$$

The jet mass measurement receives contributions from the collinear modes that pass grooming. We can now write the final form of our factorized density matrix element

$$
\Sigma^{(0)}\left(q_{T}, e_{n}, e_{\bar{n}}\right)=V \times H(Q, \mu) S\left(\vec{q}_{T} ; \mu, \nu\right) \otimes_{q_{T}} \mathcal{J}_{n}^{\perp}\left(e_{n}, Q, z_{c}, \vec{q}_{T} ; \mu, \nu\right) \otimes_{q_{T}} \mathcal{J}_{\bar{n}}^{\perp}\left(e_{\bar{n}}, Q, z_{c}, \vec{q}_{T} ; \mu, \nu\right)
$$

where $\otimes_{q_{T}}$ indicates a convolution in $\vec{q}_{T} . \mathrm{H}(\mathrm{Q})$ is the hard function which also includes the born level term. The factorized functions have both UV and rapidity divergences which are regulated by dim. reg. and a rapidity regulator $\eta$ introduced in [90] to separate the soft and jet functions which have the same virtuality. The corresponding renormalization scales are $\mu$ and $\nu$. The factorized functions are defined as follows

$$
S\left(\vec{q}_{T}\right)=\frac{1}{N_{R}} \operatorname{tr}\left\langle X_{S}\left|\mathcal{T}\left\{e^{-i \int_{0}^{\infty} d t^{\prime} H_{s, \text { int }, I}\left(t^{\prime}\right)} S_{\bar{n}}^{\dagger} S_{n}(0)\right\}\right| 0\right\rangle\left\langle 0\left|\overline{\mathcal{T}}\left\{e^{-i \int_{0}^{\infty} d t^{\prime} H_{s, \text { int }, I}\left(t^{\prime}\right)} S_{n}^{\dagger} S_{\bar{n}}(0)\right\} \delta^{2}\left(\vec{q}_{T}-\mathcal{P}_{\perp}\right)\right| X_{S}\right\rangle
$$

The trace here is a trace over color and its understood that $\left|X_{S}\right\rangle\left\langle X_{S}\right|$ includes a sum over soft states with their phase space integrated over. $\mathcal{P}_{\perp}$ action on an operator is to extract the transverse momentum $\vec{p}_{\perp}$ of the states annihilated by that operator. This computes the Soft function in a vacuum background, but as we know we actually have a background of the medium particles which also scale as the soft mode. So, in principle, we have

$$
S\left(\vec{q}_{T}\right)=\frac{1}{N_{R}} \operatorname{tr}\left\langle X_{S}\left|\mathcal{T}\left\{e^{-i \int_{0}^{\infty} d t^{\prime} H_{s, \text { int }, I}\left(t^{\prime}\right)} S_{\bar{n}}^{\dagger} S_{n}(0)\right\} \rho_{B} \overline{\mathcal{T}}\left\{e^{-i \int_{0}^{\infty} d t^{\prime} H_{s, \text { int }, I}\left(t^{\prime}\right)} S_{n}^{\dagger} S_{\bar{n}}(0)\right\} \delta^{2}\left(\vec{q}_{T}-\mathcal{P}_{\perp}\right)\right| X_{S}\right\rangle
$$

where we have assumed a time independent QGP background. Of course, we can take into account the fact that the time scale for the soft emission (which puts the collinear mode off-shell) is much shorter than the formation time for the QGP, in which case we would be justified to compute the soft function in a vacuum background, which is what we will assume for the rest of this paper. The quark jet function is defined as

$$
\begin{aligned}
& \mathcal{J}_{n}^{\perp}\left(e_{n}, Q, z_{c}, \vec{q}_{T}\right)=\frac{(2 \pi)^{3}}{N_{c}} \operatorname{tr}\left\langle X_{n}\left|\mathcal{T}\left\{e^{-i \int_{0}^{\infty} d t^{\prime} H_{n, \text { int }, I}\left(t^{\prime}\right)} \bar{\chi}_{n}(0)\right\}\right| 0\right\rangle \\
& \left\langle 0\left|\overline{\mathcal{T}}\left\{e^{-i \int_{0}^{\infty} d t^{\prime} H_{n, \text { int }, I}\left(t^{\prime}\right)} \chi_{n} \frac{\not h}{2}\right\} \delta\left(Q-\mathcal{P}^{-}\right) \delta^{2}\left(\mathcal{P}^{\perp}\right) \Theta\left(e_{n}-\mathcal{E}_{X_{n} \in \mathrm{gr}}\right) \delta^{2}\left(\vec{q}_{T}-\mathcal{P}_{X_{n} \notin \mathrm{gr}}\right)\right| X_{n}\right\rangle
\end{aligned}
$$

where there is a coloor and Dirac trace. In impact parameter space, the convolution in $\vec{q}_{T}$ turns into a product

$$
\Sigma^{(0)}=V \times H(Q, \mu) \times \int d^{2} b e^{i \vec{q}_{T} \cdot \vec{b}} S(\vec{b} ; \mu, \nu) \mathcal{J}_{n}^{\perp}\left(e_{n}, Q, z_{c}, \vec{b} ; \mu, \nu\right) \mathcal{J}_{\bar{n}}^{\perp}\left(e_{\bar{n}}, Q, z_{c}, \vec{b} ; \mu, \nu\right)
$$

where for a function $\mathcal{F}\left(\vec{q}_{T}\right)$,

$$
\mathcal{F}(\vec{b})=\int \frac{d^{2} \vec{q}_{T}}{(2 \pi)^{2}} e^{-i \vec{q}_{T} \cdot \vec{b}} \mathcal{F}\left(\vec{q}_{T}\right)
$$


The jet and soft functions then obey two renormalization group equations, one in $\mu$ and another in $\nu$.

$$
\begin{aligned}
\mu \frac{d}{d \mu} \mathcal{F}(\vec{b}, \mu, \nu) & =\gamma_{\mu}^{\mathcal{F}} \mathcal{F}(\vec{b}, \mu, \nu) \\
\nu \frac{d}{d \nu} \mathcal{F}(\vec{b}, \mu, \nu) & =\gamma_{\nu}^{\mathcal{F}} \mathcal{F}(\vec{b}, \mu, \nu)
\end{aligned}
$$

The one loop results for all the functions in $\vec{b}$ space are presented in appendix A. The one loop results reveal that each function has a natural scale for $\mu$ and $\nu$ at which the logarithms are minimized. For e.g., looking at eq. (A.3), we see that at one loop, the logarithms for the soft function are minimized at $\mu=\nu=\left(b e^{\gamma_{E}}\right)^{-1}$, while for the jet function (eq. (A.9)), the scales appear to be $\mu=\left(b e^{\gamma_{E}}\right)^{-1} \sim \sqrt{e_{n}} E_{J}, \nu=Q z_{c}$. For the hard function, we have $\mu=Q$. Solving the renormalization group equations in $\mu, \nu$ therefore allows us to resum large logarithms in the ratio of widely separated scales.

\subsection{Next-to-leading order in Glauber}

We now consider the next to leading order term in the expansion of the Glauber Hamiltonian starting from eq. (3.20). In principle, we should start off with a single insertion of $H^{G}$ on either side of the cut. Since we have a non-trivial soft function consisting of Soft Wilson lines (eq. (3.30)) at leading order which scales the same way as the soft partons of the medium, the single $H^{G}$ insertion can lead to interfering diagrams between the soft Wilson lines and the medium. However, as stated in the previous section, if the time scale for soft emissions is shorter than the QGP formation time, then we can factorize the QGP interactions of the jet from the explicit soft radiation off the quark created in the hard interaction in which case we need to do atleast a quadratic Glauber insertion. This also follows another approximation we will make in section 4 , where we retain only the diagrams where the partons created in the hard interaction go on-shell before interacting with the medium. With a quadratic Glauber insertion, we can have two contributions depending on whether the two Glauber insertions are on the same or opposite sides of the cut. This respectively corresponds to a single virtual and real interaction of the jet with the medium.

$$
\Sigma^{(2)}(t)=\Sigma_{R}^{(2)}(t)+\left\{\Sigma_{V}^{(2)}(t)+\text { c.c. }\right\}
$$

We now turn towards deriving factorization for each of these terms.

Glauber insertion on both sides of the cut. We begin with eq. (3.18) and expand to insert one $H^{G}$ factor on each side of the cut.

$$
\begin{aligned}
\sigma_{R}^{(2)}(t)= & |C(Q)|^{2} I_{\mu \nu} \int d \tilde{x} e^{-i H^{\mathrm{SCET}}} t \mathcal{T}\left\{\int_{0}^{t} d t^{\prime} H_{I_{s c}}^{G}\left(t^{\prime}\right) J_{\mathrm{SCET}, I_{s c}}^{\mu}\left(x_{1}\right)\right\}|0\rangle \\
& \langle 0| \overline{\mathcal{T}}\left\{\int_{0}^{t} d \hat{t} H_{I_{s c}}^{G}(\hat{t}) J_{\mathrm{SCET}, I_{s c}}^{\nu}\left(x_{2}\right)\right\} e^{i H^{\mathrm{SCET}} t}
\end{aligned}
$$


By following the same series of steps as for the leading order term, we can write the result in terms of the free theory interaction picture indicated henceforth by the subscript I.

$$
\begin{aligned}
\sigma_{R}^{(2)}(t)= & |C(Q)|^{2} I_{\mu \nu} \int d \tilde{x} e^{-i H^{0} t} \mathcal{T}\left\{e^{-i \int_{0}^{t} d t^{\prime} H_{\mathrm{int}, I}\left(t^{\prime}\right)} \int_{0}^{t} d t_{a} H_{I}^{G}\left(t_{a}\right) J_{\text {SCET }, I}^{\mu}\left(x_{1}\right)\right\}|0\rangle \\
& \times\langle 0| \overline{\mathcal{T}}\left\{e^{-i \int_{0}^{t} d t^{\prime} H_{\mathrm{int}, I}\left(t^{\prime}\right)} \int_{0}^{t} d t_{b} H_{I}^{G}\left(t_{b}\right) J_{\text {SCET }, I}^{\nu}\left(x_{2}\right)\right\} e^{i H^{0} t}
\end{aligned}
$$

The next step is to obtain a factorized formula in terms of our EFT modes. To do this, we explicitly put in the form of our Glauber operator, considering the case of collinear partons interacting with the soft partons in the medium. These operators were defined in eq. (3.10).

$$
H^{G}(t)=\sum_{i, j \in q, g} C_{i j}(Q) \int d^{3} \vec{x} O_{n, i}^{A}(\vec{x}, t) \frac{1}{\mathcal{P}_{\perp}^{2}} O_{S, j}^{A}(\vec{x}, t)
$$

We can now take the trace over the density matrix inserting our measurement as before.

$$
\begin{aligned}
& \left\langle X\left|\mathcal{M} \sigma_{R}^{(2)}(t \rightarrow \infty)\right| X\right\rangle \equiv \Sigma_{R}^{(2)} \\
& =|C(Q)|^{2} I_{\mu \nu} \int d \tilde{x}\left\langle X\left|\mathcal{T}\left\{e^{-i \int_{0}^{t} d t^{\prime} H_{\mathrm{int}, I}\left(t^{\prime}\right)} \int_{0}^{t} d t_{a} H_{I}^{G}\left(t_{a}\right) J_{\text {SCET }, I}^{\mu}\left(x_{1}\right)\right\}\right| 0\right\rangle \\
& \quad \times\left\langle 0\left|\overline{\mathcal{T}}\left\{e^{-i \int_{0}^{t} d t^{\prime} H_{\mathrm{int}, I}\left(t^{\prime}\right)} \int_{0}^{t} d t_{b} H_{I}^{G}\left(t_{b}\right) J_{\text {SCET }, I}^{\nu}\left(x_{2}\right)\right\} \mathcal{M}\right| X\right\rangle
\end{aligned}
$$

We can now follow the same series of steps as for the leading order term, and apply power counting to measurement functions as well as the momentum conserving $\delta$ functions based on the momentum scaling of each mode. Accordingly, we factorize the Hilbert space of the final states in terms of the momentum scaling of the modes and pull out the co-ordinate dependence of each mode by acting with the operators on the final state. This yields the following co-ordinate integrals

$$
\begin{aligned}
I= & \int d^{4} x_{1} e^{-i x_{1} \cdot\left(p_{e}+p_{\bar{e}}-p_{J n, 1}-p_{J S, 1}-p_{J \bar{n}}\right)} \int d^{4} x_{2} e^{-i x_{2} \cdot\left(p_{e}+p_{\bar{e}}-p_{J n, 2}-p_{J S, 2}-p_{J \bar{n}}\right)} \\
& \int d^{4} x_{a} e^{-i x_{a} \cdot\left(p_{G n, 1}+p_{G s, 1}\right)} \times \int d^{4} x_{b} e^{-i x_{b} \cdot\left(p_{G n, 2}+p_{G s, 2}\right)}
\end{aligned}
$$

where the subscripts $\mathrm{G}, \mathrm{J}$ tell us whether the momentum is coming from the action of the Glauber Hamiltonian fields or the SCET current respectively. To perform the integrals we take the limit $t \rightarrow \infty$. This means that we are making the assumption that the time of propagation of the jet in the medium or equivalently the length of medium seen by the jet is larger than all other time scales in problem. So this is the limit of a long lived medium. This may not always be a realistic assumption, especially in the case of energetic jets where the jet formation time $t_{F} \sim Q / q_{T}^{2}$ can be of the order or longer than the medium lifetime. However, this a good first step and the case of short lived medium will be considered in an upcoming paper [91]. Now performing the integrals and applying power counting, we have

$$
\begin{aligned}
I= & \delta\left(Q-p_{J n 1}^{-}\right) \delta\left(Q-p_{\bar{n}}^{+}\right) \delta^{2}\left(p_{J n, 1}^{\perp}+p_{J s, 1}^{\perp}+p_{\bar{n}}^{\perp}\right) \\
& \delta\left(Q-p_{J n 2}^{-}\right) \delta\left(Q-p_{\bar{n}}^{+}\right) \delta^{2}\left(p_{J n, 2}^{\perp}+p_{J s, 2}^{\perp}+p_{\bar{n}}^{\perp}\right) \\
& \delta\left(p_{G n, 1}^{-}\right) \delta\left(p_{G S, 1}^{+}+p_{G n, 1}^{+}\right) \delta^{2}\left(p_{G n, 1}^{\perp}+p_{G S, 1}^{\perp}\right) \\
& \delta\left(p_{G n, 2}^{-}\right) \delta\left(p_{G S, 2}^{+}+p_{G n, 2}^{+}\right) \delta^{2}\left(p_{G n, 2}^{\perp}+p_{G S, 2}^{\perp}\right)
\end{aligned}
$$


This simplification follows from the idea that $p_{G S}$ scales as the Glauber momentum since we are interested in a $\mathrm{t}$ channel exchange between the soft and collinear modes. This allows us to drop the contribution $p_{G S}^{-}$from the conservation of "-" momentum while retaining it for the "+" and $\perp$ components. We have ignored any factors of $2 \pi$ which will be absorbed in the overall co-efficient for $\Sigma_{R}^{(2)}$. We also have additional constraints since the total momentum for a particular mode must match on both sides of the cut

$$
\begin{aligned}
& p_{J n, 1}+p_{G n, 1}=p_{J n, 2}+p_{G n, 2} \\
& p_{J S, 1}+p_{G S, 1}=p_{J S, 2}+p_{G S, 2}
\end{aligned}
$$

We can simplify our measurement $\delta$ functions using these set of constraints

$$
\begin{aligned}
\delta^{2}\left(\vec{q}_{T}-p_{X_{n}, \in \mathrm{gr}}^{\perp}-p_{X_{\bar{n}}, \in \mathrm{gr}}^{\perp}\right) & \equiv \delta^{2}\left(\vec{q}_{T}-p_{X_{n}, \in \mathrm{gr}}^{\perp}-p_{\bar{X}_{\bar{n}}, \in \mathrm{gr}}^{\perp}\right) \\
& =\delta^{2}\left(\vec{q}_{T}-p_{G n, 1 \in \mathrm{gr}}^{\perp}-p_{J_{n, 1 \in \mathrm{gr}}}^{\perp}-p_{\bar{X}_{\bar{n}}, \in \mathrm{gr}}^{\perp}\right) \\
& =\delta^{2}\left(\vec{q}_{T}+\left[p_{G S, 1}^{\perp}+p_{J_{S, 1}}^{\perp}\right]+p_{J n, 1 \notin \mathrm{gr}}^{\perp}+p_{\bar{n}, \notin \mathrm{gr}}^{\perp}\right)
\end{aligned}
$$

where the term inside the square brackets is the total contribution from the Soft sector, which includes both the vacuum as well as medium effects. We have written the total contribution from the $\mathrm{n}$ collinear sector $p_{X_{n}, \in \mathrm{gr}}^{\perp}=p_{G n, 1 \in \mathrm{gr}}^{\perp}+p_{J n, 1 \in \mathrm{gr}}^{\perp}$. As for the leading order term, we can set the axis of the $\bar{n}$ jet to be exactly aligned with the $\bar{n}$ axis. Then using RPI 1 symmetry of SCET as before, we do the same for the $\mathrm{n}$ jet before it interacts with the medium. Using the rest of the constraints then, once again we have an overall $4 \mathrm{~d}$ volume factor of $V$. Since we are ignoring interference between the soft operators of the SCET and the Glauber insertion, we can set

$$
p_{G S, 1}=p_{G S, 2} \equiv p_{G S}, \quad \text { so that } p_{J S, 1}=p_{J S, 2} .
$$

We can now write down our factorization formula for $\Sigma_{R}^{(2)}$, explicitly pulling out the vacuum Soft function,

$$
\begin{aligned}
\Sigma_{R}^{(2)}= & V \times\left|C_{G}\right|^{2} H(Q, \mu)\left\{\int d^{2} \vec{q}_{J S} S\left(\vec{q}_{J S}\right)\right\}\left\{\int d^{2} \vec{q}_{G S} d p_{G S}^{+} S_{G}^{A B}\left(q_{G S}^{\perp}, p_{G S}^{+}\right)\right\} \\
& \left\{\int d^{2} \vec{q}_{T n} \int d^{2} \vec{p}_{G n, 1}^{\perp} d^{2} \vec{p}_{G n, 2}^{\perp} d p_{G n, 1}^{+} d p_{G n, 2}^{+} J_{n}^{A B}\left(e_{n}, \vec{p}_{G n, 1}^{\perp}, \vec{p}_{G n, 2}^{\perp}, p_{G n, 1}^{+}, p_{G n, 2}^{+}, \vec{q}_{T n}\right)\right\} \\
& \times \int d^{2} \vec{q}_{T \bar{n}} J_{\bar{n}}\left(e_{\bar{n}}, \vec{q}_{\bar{n}}\right) \delta^{2}\left(\vec{q}_{T}+\vec{q}_{J S}+\vec{q}_{G S}+\vec{q}_{T n}+\vec{q}_{T \bar{n}}\right) \\
& \times \delta^{2}\left(\vec{q}_{G S}+\vec{p}_{G n, 1}^{\perp}\right) \delta^{2}\left(\vec{q}_{G S}+\vec{p}_{G n, 2}^{\perp}\right) \delta\left(p_{G S}^{+}+p_{G n, 1}^{+}\right) \delta\left(p_{G S}^{+}+p_{G n, 2}^{+}\right)
\end{aligned}
$$

In order to simplify notation, its easiest to express this result by rewriting some of the momentum conserving $\delta$ functions in co-ordinate space. This gives us

$$
\begin{aligned}
\Sigma_{R}^{(2)}= & V \times\left|C_{G}\right|^{2} H(Q, \mu)\left\{\int d^{2} \vec{q}_{J S} S\left(\vec{q}_{J S}\right)\right\} \\
& \times \int d^{4} x \int d^{4} y\left\{\int d^{2} \vec{q}_{G S} \bar{S}_{G}^{A B}\left(q_{G S}^{\perp},\left\{x_{\perp}, x^{-}\right\},\left\{y_{\perp}, y^{-}\right\}\right)\right\}\left\{\int d^{2} \vec{q}_{T n} J_{n}^{A B}\left(e_{n}, x, y, \vec{q}_{T n}\right)\right\} \\
& \int d^{2} \vec{q}_{\bar{n}} \mathcal{J}_{\bar{n}}^{\perp}\left(e_{\bar{n}}, \vec{q}_{T \bar{n}}\right) \delta^{2}\left(\vec{q}_{T}+\vec{q}_{J S}+\vec{q}_{G S}+\vec{q}_{T n}+\vec{q}_{T \bar{n}}\right)
\end{aligned}
$$


While most of the functions remain unchanged compared to their vacuum counterparts, we have two new/modified function $\bar{S}_{G}^{A B}$ and $J_{n}^{A B}$ defined as

$$
\begin{aligned}
& \bar{S}_{G}^{A B}\left(q_{G S}^{\perp},\left\{x_{\perp}, x^{-}\right\},\left\{y_{\perp}, y^{-}\right\}\right)=\left\langle X_{S}\left|\left\{\delta^{2}\left(q_{G S}^{\perp}-\mathcal{P}_{\perp}\right) \frac{1}{\mathcal{P}_{\perp}^{2}} O_{S}^{A}(\hat{x})\right\} \rho_{B} \frac{1}{\mathcal{P}_{\perp}^{2}} O_{S}^{B}(\hat{y})\right| X_{S}\right\rangle \\
& J_{n}^{A B}\left(e_{n}, x, y\right)=\left\langle X_{n}\left|T\left\{\bar{\chi}_{n}(0) \frac{\hbar}{2} O_{n}^{A}(x)\right\}\right| 0\right\rangle\left\langle 0\left|\bar{T}\left\{O_{n}^{B}(y)\left[\delta^{2}\left(\mathcal{P}_{\perp}\right) \chi_{n}(0)\right]\right\} \delta^{2}\left(Q-\mathcal{P}^{-}\right) \mathcal{M}_{n}\right| X_{n}\right\rangle
\end{aligned}
$$

where for the rest of the paper we use $\hat{x} \equiv\left(x^{-}, 0, \vec{x}_{\perp}\right)$ and likewise $\hat{y}$, and

$$
\mathcal{M}_{n}=\Theta\left(e_{n}-\mathcal{E}_{X_{n} \in \mathrm{gr}}\right) \delta^{2}\left(\vec{q}_{T n}-\mathcal{P}_{X_{n} \notin \mathrm{gr}}^{\perp}\right)
$$

To derive the factorization formula, we have used the idea that the Wilson coefficient $C_{G}$ for all the Glauber operators is identical,

$$
C_{G}=8 \pi \alpha_{s}
$$

and

$$
O_{n}^{A}=\sum_{i} O_{n, i}^{A}, \quad O_{S}^{B}=\sum_{j} O_{S, j}^{B}
$$

Using the translational invariance of the QGP medium we can write

$$
\bar{S}_{G}^{A B}\left(q_{G S}^{\perp}, \hat{x}, \hat{y}\right)=\int \frac{d^{4} k}{(2 \pi)^{4} k_{\perp}^{4}} e^{i(\hat{x}-\hat{y}) \cdot k} D_{>}^{A B}(k) \delta^{2}\left(q_{G S}^{\perp}-\vec{k}_{\perp}\right)
$$

where $D_{>}^{A B}(k)$ is the Soft correlator in the medium. The assumption of translational invariance is an idealized scenario, but it is not a necessary condition for factorization. Since a single interaction of the jet with the medium is instantaneous and probes it over length scales inverse of the transverse momentum exchanged $\sim 1 / k_{\perp}$, the factorization is robust against inhomogeneties in the medium over length scales $\gg 1 / k_{\perp} \sim 1 / T$, i.e. as long as the jet sees a uniform medium over the length-time scales of a single Glauber exchange. The factorization formula incorporating such inhomogeneties will be considered in an upcoming paper [91].

$$
D_{>}^{A B}(k)=\int d^{4} x e^{-i k \cdot x}\left\langle X_{S}\left|O_{S}^{A}(x) \rho O_{S}^{B}(0)\right| X_{S}\right\rangle
$$

We note here that this function is independent of $k^{+}$.

We can redefine $\vec{q}_{n} \rightarrow \vec{q}_{n}-\vec{k}_{\perp}$, and write a compact formula

$$
\begin{aligned}
\Sigma_{R}^{(2)}\left(\vec{q}_{T}, e_{n}, e \bar{n}\right)= & V \times\left|C_{G}\right|^{2} H(Q, \mu) S\left(\vec{q}_{T}\right) \otimes_{q_{T}} \mathcal{J}_{\bar{n}}^{\perp}\left(e_{\bar{n}}, \vec{q}_{T}\right) \\
& \otimes_{q_{T}} \int \frac{d^{2} k_{\perp} d k^{-}}{k_{\perp}^{4}} D_{>}^{A B}\left(k_{\perp}, k^{-}\right) \mathcal{J}_{n}^{A B}\left(e_{n}, \vec{q}_{T}, \vec{k}_{\perp}\right)
\end{aligned}
$$

where we have

$$
\mathcal{J}_{n}^{A B}\left(e_{n}, \vec{q}_{T}, \vec{k}_{\perp}\right)=\int d k^{+} \int d^{4} x \int d^{4} y e^{i(\hat{x}-\hat{y}) \cdot k} J_{n}^{A B}\left(e_{n}, x, y, \vec{q}_{T}-\vec{k}_{\perp}\right)
$$


The convolution in $\vec{q}_{T}$ turns into a product in impact parameter space. We can now divide and multiply by the vacuum jet function $\mathcal{J}_{n}^{\perp}\left(e_{n}, \vec{b}\right)$ which allows us to factor out the vacuum cross section and define a medium structure function and a medium induced jet function

$$
\Sigma_{R}^{(2)}\left(\vec{q}_{T}, e_{n}, e_{\bar{n}}\right)=\left|C_{G}\right|^{2} \int d^{2} \vec{b} e^{i \vec{b} \cdot \vec{q}_{T}} \Sigma^{(0)}\left(b, e_{n}, e_{\bar{n}}\right) \int d^{2} k_{\perp} S_{G}^{A B}\left(k_{\perp}\right) J_{n, M}^{A B}\left(e_{n}, \vec{b}, \vec{k}_{\perp}\right)
$$

where

$$
\begin{aligned}
J_{n, M}^{A B}\left(e_{n}, \vec{b}, \vec{k}_{\perp}\right) & =\frac{1}{k_{\perp}^{2}} \frac{\mathcal{J}_{n}^{A B}\left(e_{n}, \vec{b}, \vec{k}_{\perp}\right)}{\mathcal{J}_{n}^{\perp}\left(e_{n}, \vec{b}\right)} \\
S_{G}^{A B}\left(k_{\perp}\right) & =\int d k^{-} \frac{1}{k_{\perp}^{2}} D_{>}^{A B}\left(k_{\perp}, k^{-}\right)
\end{aligned}
$$

Glauber insertion on the same side of the cut. We can now look at the piece we get by doing two Glauber insertions on the same side of the cut starting from eq. (3.20). Here, I will consider inserting on the bra side and deal with the ket in the same manner later on.

$$
\begin{aligned}
\sigma_{V}^{(2)}(t)= & \frac{(-i)^{2}}{2} I_{\mu \nu}|C(Q)|^{2} \int d \tilde{x} e^{-i H^{\mathrm{SCET}}} t \mathcal{T}\left\{\int_{0}^{t} d t^{\prime} H_{I_{s c}}^{G}\left(t^{\prime}\right) \int_{0}^{t} d \hat{t} H_{I_{s c}}^{G}(\hat{t}) J_{\mathrm{SCET}, I_{s c}}^{\mu}\left(x_{1}\right)\right\}|0\rangle \\
& \langle 0| \overline{\mathcal{T}}\left\{J_{\mathrm{SCET}, I_{s c}}^{\nu}\left(x_{2}\right)\right\} e^{i H^{\mathrm{SCET}} t}
\end{aligned}
$$

Following the same series of steps as in the previous section, we arrive at a similar factorization formula

$$
\begin{aligned}
\Sigma_{V}^{(2)}= & -\frac{1}{2} V \times\left|C_{G}(Q)\right|^{2} H(Q, \mu) \int d^{2} \vec{q}_{J S} S\left(\vec{q}_{J S}\right) \int d^{2} \vec{q}_{T \bar{n}} \mathcal{J}_{\bar{n}}^{\perp}\left(e_{\bar{n}}, \vec{q}_{T \bar{n}}\right) \\
& \int d^{4} x \int d^{4} y \bar{S}_{G}^{A B}(\hat{x}, \hat{y}) \int d^{2} \vec{q}_{T n} J_{n}^{A B}\left(e_{n}, \vec{q}_{T n}, x, y\right) \delta^{2}\left(\vec{q}_{T n}+\vec{q}_{J S}+\vec{q}_{T n}+\vec{q}_{T \bar{n}}\right)
\end{aligned}
$$

with the following definitions

$$
\begin{gathered}
\bar{S}_{G}^{A B}(\hat{x}, \hat{y})=\left\langle X_{S}\left|\mathcal{T}\left\{O_{S}^{A}(\hat{x}) O_{S}^{B}(\hat{y})\right\} \rho_{B}\right| X_{S}\right\rangle \\
J_{n}^{A B}\left(e_{n}, \vec{q}_{T n}, x, y\right)=\left\langle X_{n}\left|\mathcal{T}\left\{\bar{\chi}_{n}(0) \frac{\not h}{2} O_{n}^{A}(x) O_{n}^{B}(y)\right\}\right| 0\right\rangle\left\langle 0\left|\left[\delta^{2}\left(\mathcal{P}_{\perp}\right) \chi_{n}(0)\right] \delta^{2}\left(Q-\mathcal{P}^{-}\right) \mathcal{M}_{n}\right| X_{n}\right\rangle
\end{gathered}
$$

The term with Glauber insertions on the other side of the cut can now be trivially obtained from this result.

\section{Decoherence of the hard interaction from medium scattering in a long lived medium}

One aspect of this factorization which is different compared to a vacuum factorization result is the presence of the environment. Since the QGP medium in not coherently connected with the hard interaction that produces the jet, the phase space of the jet allows the partons to go on-shell before they interact with the medium. In fact, the most dominant contribution 
to the cross section comes from this region of phase space. This is only true if there is enough time for the partons created in the jet to go on-shell which happens when the time of jet propagation in the medium $t_{M}$ is much greater than the formation time of the jet $t_{F} \sim Q / q_{T}^{2}$. As already alluded to earlier, this is the case of a long-lived medium. This may not always be a realistic scenario especially for the case of high energy jets which have a long formation time. In this case quantum interference between the hard interaction and the medium interaction becomes important. This is a more involved case and I will detail in section 8 how this scenario, which will be considered in an upcoming paper [91], will modify the form of factorization.

Coming back to the case of a long lived medium, we can look at the tree level result for our modified jet function defined in eq. (3.42) that appears in the factorized formula for $\Sigma_{R}^{(2)}$ in eq. (3.48)

$$
\begin{aligned}
\mathcal{J}_{n}^{A B(0)}= & \int d k^{+} \int d^{4} x \int d^{4} y e^{-i k \cdot(\hat{x}-\hat{y})} e^{i p \cdot(x-y)} \int \tilde{d} p \operatorname{Tr}\left[\bar{u}(p) T^{A} \frac{\not h}{2} D(x) \frac{\not h}{2} D^{\dagger}(y) \frac{\not h}{2} T^{B} u(p)\right] \\
& \delta^{2}\left(p_{\perp}\right) \delta\left(Q-p^{-}\right) \delta^{2}\left(\vec{q}_{n}-\vec{k}_{\perp}\right) \\
= & \int d k^{+} \int d^{4} x \int d^{4} y e^{-i k \cdot(\hat{x}-\hat{y})} e^{i p \cdot(x-y)} \int \tilde{d} p \\
& \operatorname{Tr}\left[\bar{u}(p) T^{A} \frac{\not h}{2} \int d^{4} q \frac{\not h}{2} \frac{q^{-} e^{-i q \cdot x}}{q^{2}-i \epsilon} \frac{\not h}{2} \int d^{4} q^{\prime} \frac{h}{2} \frac{\left(q^{\prime}\right)^{-} e^{i q^{\prime} \cdot y}}{\left(q^{\prime}\right)^{2}+i \epsilon} \frac{\not h}{2} T^{B} u(p)\right] \delta^{2}\left(p_{\perp}\right) \delta\left(Q-p^{-}\right) \delta^{2}\left(\vec{q}_{n}-\vec{k}_{\perp}\right)
\end{aligned}
$$

where henceforth we will reserve the notation $\tilde{d p}$ to mean the integral over the phase space of the on-shell massless particle $\mathrm{p}$

$$
\tilde{d} p=\frac{d^{3} p}{2 E_{p}} \equiv d^{4} p \delta^{+}\left(p^{2}\right)
$$

The integrals over the co-ordinates $\mathrm{x}$ and y now sets $q=q^{\prime}=p+k$. Since we are integrating over $\mathrm{k}$, we see that there is a pinch singularity in the integral over $k^{+}$and the dominant contribution comes from the region when the intermediate propagators (in $q=q^{\prime}$ ) go on-shell. So we can replace the propagators with their on-shell (cut) forms. The pinch singularity seems to yield a divergence. However, we notice that the integrals over $d x^{0}, d y^{0}$ are actually over a finite time interval $\in\{0, t\}$ and hence, what naively appears as an infinite result actually yields a factor of $t$, which is just the time of propagation of the jet in the medium. We are effectively expanding in $t_{i} / t$, where $t_{i}$ are all other time scales in the problem, namely the formation time of the soft and collinear radiation.

We can therefore revisit our factorized functions and simplify them by looking at this dominant contribution where the intermediate partons produced in the hard interaction go on-shell before interacting with the medium one at a time. This will capture all the corrections enhanced by a factor of $t$, which will be the dominant correction.

$\boldsymbol{\Sigma}_{\boldsymbol{R}}^{(2)}$. The medium induced jet function is defined in eq. (3.51) as

$$
J_{n, M}^{A B}\left(e_{n}, \vec{b}, \vec{k}_{\perp}\right)=\frac{1}{k_{\perp}^{2}} \frac{\mathcal{J}_{n}^{A B}\left(e_{n}, \vec{b}, \vec{k}_{\perp}\right)}{\mathcal{J}_{n}^{\perp}\left(e_{n}, \vec{b}\right)}
$$


Since we are working in a regime where the intermediate partons are going on-shell, we can do a further factorization of the jet function to separate out the Glauber and vacuum terms explicitly. At the same time, in anticipation of the fact that the jet function will give a result proportional to $t$, we can define

$$
\begin{aligned}
\mathcal{J}_{n, M}^{A B}\left(e_{n}, k_{\perp}, b\right)= & \frac{1}{k_{\perp}^{2} t \mathcal{J}_{n}^{\perp}\left(e_{n}, \vec{b}\right)} \int \frac{d^{2} q_{n} e^{i \vec{q}_{n} \cdot \vec{b}}}{(2 \pi)^{2}} \int d k^{+} \int d^{4} x \int d^{4} y e^{-i k \cdot(\hat{x}-\hat{y})} \\
& \times\left\langle X_{n}\left|O_{n}^{A}(x) \delta^{2}\left(\mathcal{P}^{\perp}\right) \delta\left(Q-p^{-}\right) \chi_{n}(0) \bar{\chi} \frac{\not h}{2}\right| 0\right\rangle\left\langle 0\left|\chi_{n}(0) O_{n}^{B}(y) \mathcal{M}\right| X_{n}\right\rangle
\end{aligned}
$$

where

$$
\mathcal{M}=\Theta\left(e_{n}-\mathcal{E}_{X_{n} \in \mathrm{gr}}\right) \delta^{2}\left(\vec{q}_{n}-\mathcal{P}_{X_{n} \notin \mathrm{gr}}^{\perp}-\vec{k}_{\perp}\right)
$$

and we have dropped the time ordering between the SCET and Glauber currents. We look at the numerator(sans the Fourier transform) and we can insert a complete set of on-shell states separating the hard and Glauber operator, thus placing the intermediate partons on-shell.

$$
\begin{aligned}
\mathcal{J}^{A B}\left(e_{n}, k_{\perp}, \vec{q}_{n}\right)= & \int d k^{+} \int d^{4} x \int d^{4} y e^{-i k \cdot(\hat{x}-\hat{y})}\left\langle X_{n}\left|O_{n}^{A}(x)\right| Y_{n}\right\rangle \\
& \left\langle Y_{n}\left|\delta^{2}\left(\mathcal{P}_{\perp}\right) \delta\left(Q-p^{-}\right) \chi_{n}(0) \bar{\chi} \frac{\hbar}{2}\right| 0\right\rangle\left\langle 0\left|\chi_{n}(0)\right| \tilde{Y}_{n}\right\rangle\left\langle\tilde{Y}_{n}\left|O_{n}^{B}(y) \mathcal{M}\right| X_{n}\right\rangle
\end{aligned}
$$

where its understood that there is an integral over the phase space of all the inserted states $Y_{n}, \tilde{Y}_{n}$ and the measurement $\mathcal{M}$ acts on all final state $X_{n}$. We can now perform the co-ordinate integrals to give

$$
\begin{aligned}
& \mathcal{J}^{A B}\left(e_{n}, k_{\perp}, \vec{q}_{n}\right) \\
& =\delta\left(p_{X}^{-}-p_{Y}^{-}\right) \delta\left(p_{\tilde{Y}}^{+}-p_{Y}^{+}\right) \delta^{2}\left(k_{\perp}+p_{Y}^{\perp}-p_{X}^{\perp}\right) \delta\left(p_{X}^{-}-p_{\tilde{Y}}^{-}\right) \delta^{2}\left(k_{\perp}+p_{\tilde{Y}}^{\perp}-p_{X}^{\perp}\right) \\
& \quad\left\langle X_{n}\left|O_{n}^{A}(0)\right| Y_{n}\right\rangle\left\langle\tilde{Y}_{n}\left|O_{n}^{B}(0) \mathcal{M}\right| X_{n}\right\rangle\left\langle Y_{n}\left|\delta^{2}\left(\mathcal{P}_{\perp}\right) \delta\left(Q-p^{-}\right) \bar{\chi}_{n}(0) \frac{\not h}{2}\right| 0\right\rangle\left\langle 0\left|\chi_{n}(0)\right| \tilde{Y}_{n}\right\rangle \mathcal{M}_{X_{n}}
\end{aligned}
$$

The $\delta$ functions then imply $p_{Y}=p_{\tilde{Y}}$, where $p_{Y}=\sum_{i} p_{Y i}$ is the sum of momentum of the $Y_{n}$ states and so on.

The terms proportional to $t$ are obtained from those diagrams where one of the partons created in the hard vertex interacts with the medium. We can therefore write $\left|Y_{n}\right\rangle \equiv\left|Y_{1}\right\rangle\left|\mathcal{Y}_{n}\right\rangle$ where we have explicitly separated out the parton with momentum $p_{Y_{1}}$ which will interact with the medium. Likewise $\left|\tilde{Y}_{n}\right\rangle \equiv\left|\tilde{Y}_{1}\right\rangle\left|\tilde{\mathcal{Y}}_{n}\right\rangle$. The partons with total momenta $p \mathcal{Y}, p_{\tilde{\mathcal{Y}}_{n}}$ do not interact with the medium. At the same time, we need to drop any interference diagrams between the hard vertex and Glauber vertex to retain the t enhanced terms which allows us to write

$$
\begin{aligned}
\mathcal{J}^{A B}\left(e_{n}, k_{\perp}, \vec{q}_{n}\right)= & \delta\left(p_{X}^{-}-p_{Y 1}^{-}-p_{\mathcal{Y}}^{-}\right) \delta\left(p_{\tilde{Y}_{1}}^{+}+p_{\tilde{\mathcal{Y}}}^{+}-p_{Y_{1}}^{+}-p_{\mathcal{Y}}^{+}\right) \delta^{2}\left(k_{\perp}+p_{Y_{1}}^{\perp}+p_{\mathcal{Y}}^{\perp}-p_{X}^{\perp}\right) \\
& \delta\left(p_{X}^{-}-p_{\tilde{Y}_{1}}^{-}-p_{\tilde{\mathcal{Y}}}^{-}\right) \delta^{2}\left(k_{\perp}+p_{\tilde{Y} 1}^{\perp}+p_{\tilde{\mathcal{Y}}}^{\perp}-p_{X}^{\perp}\right)\left\langle\tilde{X}_{n}\left|O_{n}^{A}(0)\right| Y_{1}\right\rangle\left\langle\tilde{Y}_{1}\left|O_{n}^{B}(0) \mathcal{M}\right| \tilde{X}_{n}\right\rangle \\
& \times\left\langle Y_{1} \mathcal{Y}\left|\delta^{2}\left(\mathcal{P}^{\perp}\right) \delta\left(Q-p^{-}\right) \bar{\chi}_{n}(0) \frac{\not h}{2}\right| 0\right\rangle\left\langle 0\left|\chi_{n}(0)\right| \tilde{Y}_{1} \tilde{\mathcal{Y}}\right\rangle \mathcal{M}_{X_{n}}\left\langle\bar{X}_{n} \mid \mathcal{Y}\right\rangle\left\langle\tilde{\mathcal{Y}} \mid \bar{X}_{n}\right\rangle
\end{aligned}
$$


where we have split $\left|X_{n}\right\rangle \equiv\left|\bar{X}_{n}\right\rangle\left|\tilde{X}_{n}\right\rangle$ We can now eliminate the phase space integrals over all the partons that make up $p_{\mathcal{Y}}$ and $p_{\tilde{\mathcal{Y}}}$

$$
\begin{aligned}
& \mathcal{J}^{A B}\left(e_{n}, k_{\perp}, \vec{q}_{n}\right) \\
& =\delta\left(p_{\tilde{X}}^{-}-p_{Y_{1}}^{-}\right) \delta\left(p_{\tilde{Y}_{1}}^{+}-p_{Y_{1}}^{+}\right) \delta^{2}\left(k_{\perp}+p_{Y_{1}}^{\perp}-p_{\tilde{X}}^{\perp}\right) \delta\left(p_{\tilde{X}}^{-}-p_{\tilde{Y}_{1}}^{-}\right) \delta^{2}\left(k_{\perp}+p_{\tilde{Y} 1}^{\perp}-p_{\tilde{X}}^{\perp}\right) \\
& \quad\left\langle\tilde{X}_{n}\left|O_{n}^{A}(0)\right| Y_{1}\right\rangle\left\langle\tilde{Y}_{1}\left|O_{n}^{B}(0) \mathcal{M}\right| \tilde{X}_{n}\right\rangle\left\langle Y_{1} \bar{X}_{n}\left|\delta^{2}\left(\mathcal{P}^{\perp}\right) \delta\left(Q-p^{-}\right) \bar{\chi}_{n}(0) \frac{\not h}{2}\right| 0\right\rangle\left\langle 0\left|\chi_{n}(0)\right| \tilde{Y}_{1} \bar{X}_{n}\right\rangle \mathcal{M}_{X_{n}}
\end{aligned}
$$

which tells us that $p_{Y 1}=p_{\tilde{Y} 1}=p_{X}-p_{\bar{X}}-k=p_{\tilde{X}}-k$. We can now perform the phase space integrals over $p_{Y 1}, p_{\tilde{Y} 1}$, which yields a redundant $\delta$ function which is just the factor of $t$ that we expected. We can therefore write

$$
\begin{aligned}
\mathcal{J}^{A B}\left(e_{n}, k_{\perp}, \vec{q}_{n}\right)= & \frac{t}{\left[p_{\tilde{X}}^{-}\right]^{2}}\left\langle p_{\tilde{X}}-k, \bar{X}_{n}\left|\delta^{2}\left(\mathcal{P}_{\perp}\right) \delta\left(Q-p^{-}\right) \bar{\chi}_{n}(0) \frac{\hbar \hbar}{2}\right| 0\right\rangle\left\langle 0\left|\chi_{n}(0)\right| p_{\tilde{X}}-k, \bar{X}_{n}\right\rangle \\
& \times\left\langle\tilde{X}_{n}\left|O_{n}^{A}(0)\right| p_{\tilde{X}}-k\right\rangle\left\langle p_{\tilde{X}}-k\left|O_{n}^{B}(0)\right| \tilde{X}_{n}\right\rangle \mathcal{M}_{X_{n}}
\end{aligned}
$$

which allows us to rewrite our factorization formula for $\Sigma_{R}^{(2)}$ from eq. (3.50) as

$$
\Sigma_{R}^{(2)}\left(\vec{q}_{T}, e_{n}, e_{\bar{n}}\right)=t\left|C_{G}\right|^{2} \int d^{2} b e^{i \vec{q}_{T} \cdot \vec{b}} \Sigma^{(0)}\left(\vec{b}, e_{n}, e_{\bar{n}}\right) \int d^{2} k_{\perp} S_{G}^{A B}\left(k_{\perp}\right) \mathcal{J}_{n, R}^{A B}\left(e_{n}, b, \vec{k}_{\perp}\right)
$$

with

$$
\begin{aligned}
\mathcal{J}_{n, R}^{A B}\left(e_{n}, \vec{q}_{T}, \vec{k}_{\perp}\right)= & \frac{1}{\mathcal{J}_{n}^{\perp}\left(e_{n}, b\right) k_{\perp}^{2}} \frac{1}{\left[p_{\tilde{X}}^{-}\right]^{2}} \int \frac{d^{2} q_{n} e^{-i \vec{q}_{n} \cdot \vec{b}}}{(2 \pi)^{2}}\left\langle p_{\tilde{X}}-k, \bar{X}_{n}\left|\delta^{2}\left(\mathcal{P}_{\perp}\right) \delta\left(Q-p^{-}\right) \bar{\chi}_{n}(0) \frac{\not h}{2}\right| 0\right\rangle \\
& \times\left\langle 0\left|\chi_{n}(0)\right| p_{\tilde{X}}-k, \bar{X}_{n}\right\rangle\left\langle\tilde{X}_{n}\left|O_{n}^{A}(0)\right| p_{\tilde{X}}-k\right\rangle\left\langle p_{\tilde{X}}-k\left|O_{n}^{B}(0)\right| \tilde{X}_{n}\right\rangle \mathcal{M}_{X_{n}}
\end{aligned}
$$

$\boldsymbol{\Sigma}_{\boldsymbol{V}}^{(2)}$. We can now do a similar simplification for the virtual piece. The factorized form for this piece was derived in eq. (3.52) in terms of the functions $S_{G}$ convoluted with a jet function with the following definitions

$$
\begin{gathered}
\bar{S}_{G}^{A B}(\hat{x}, \hat{y})=\left\langle X_{S}\left|T\left\{\frac{1}{\mathcal{P}_{\perp}^{2}} O_{S}^{A}(\hat{x}) \frac{1}{\mathcal{P}_{\perp}^{2}} O_{S}^{B}(\hat{y})\right\} \rho_{B}\right| X_{S}\right\rangle \\
J_{n}^{A B}\left(e_{n}, \vec{q}_{n}, x, y\right)=\left\langle X_{n}\left|\mathcal{T}\left\{\chi_{n}(0) \frac{\not h}{2} O_{n}^{A}(x) O_{n}^{B}(y)\right\}\right| 0\right\rangle\left\langle 0\left|\overline{\mathcal{T}}\left\{\bar{\chi}_{n}(0)\right\} \delta^{2}\left(\mathcal{P}_{\perp}\right) \delta^{2}\left(Q-\mathcal{P}^{-}\right) \mathcal{M}_{V}\right| X_{n}\right\rangle
\end{gathered}
$$

where

$$
\mathcal{M}_{V}=\Theta\left(e_{n}-\mathcal{E}_{X_{n} \in \mathrm{gr}}\right) \delta^{2}\left(\vec{q}_{n}-\mathcal{P}_{X_{n} \notin \mathrm{gr}}^{\perp}\right)
$$

Once again we are going to put the intermediate particles on-shell before they interact with the medium. This allows us to simplify our jet function by inserting a complete basis on on-shell states

$$
J_{n}^{A B}(x, y)=\left\langle X_{n}\left|\mathcal{T}\left\{O_{n}^{A}(x) O_{n}^{B}(y)\right\}\right| Y_{n}\right\rangle\left\langle Y_{n}\left|\chi_{n}(0) \frac{\not h}{2}\right| 0\right\rangle\left\langle 0\left|\bar{\chi}_{n}(0) \delta^{2}\left(\mathcal{P}_{\perp}\right) \delta^{2}\left(Q-\mathcal{P}^{-}\right) \mathcal{M}_{V}\right| X_{n}\right\rangle
$$


Following the same logic as for $\Sigma_{R}^{(2)}$, we look at the scenario where a single parton created in the hard vertex $\left(Y_{1}\right)$ interacts with the medium at a time ignoring any interference diagrams between the two vertices.

$$
\begin{aligned}
J_{n}^{A B}(x, y)= & \left\langle\tilde{X}_{n}\left|\mathcal{T}\left\{O_{n}^{A}(x) O_{n}^{B}(y)\right\}\right| Y_{1}\right\rangle\left\langle Y_{1} \mathcal{Y}\left|\chi_{n}(0) \frac{\not h}{2}\right| 0\right\rangle \\
& \times\left\langle 0\left|\bar{\chi}_{n}(0) \delta^{2}\left(\mathcal{P}_{\perp}\right) \delta^{2}\left(Q-\mathcal{P}^{-}\right) \mathcal{M}_{V}\right| \tilde{X}_{n} \bar{X}_{n}\right\rangle\left\langle\bar{X}_{n} \mid \mathcal{Y}\right\rangle
\end{aligned}
$$

which allows us to eliminate the phase space integrals over $\mathcal{Y}$. Once again we have split $\left.\left|X_{n}\right\rangle \equiv\left|\bar{X}_{n}\right\rangle \tilde{X}_{n}\right\rangle$. At the same time since we are ignoring interference diagrams between the hard and Glauber vertices, this necessarily means that $\tilde{X}_{n}$ is a single particle state.

$$
\begin{aligned}
J_{n}^{A B}(x, y)= & \left\langle\tilde{X}_{n}\left|\mathcal{T}\left\{O_{n}^{A}(x) O_{n}^{B}(y)\right\}\right| Y_{1}\right\rangle\left\langle Y_{1} \bar{X}_{n}\left|\chi_{n}(0) \frac{\not h}{2}\right| 0\right\rangle \\
& \left\langle 0\left|\bar{\chi}_{n}(0) \delta^{2}\left(\mathcal{P}_{\perp}\right) \delta^{2}\left(Q-\mathcal{P}^{-}\right) \mathcal{M}_{V}\right| \tilde{X}_{n} \bar{X}_{n}\right\rangle
\end{aligned}
$$

We can now look at the combination of the Soft and jet function and completely separate out the medium dependent piece

$$
\begin{aligned}
\mathcal{G}= & \int d^{4} x \int d^{4} y S_{G}^{A B}(\hat{x}, \hat{y}) J_{n}^{A B}(x, y) \\
= & 2 \int d^{4} x \int d^{4} y \Theta\left(x^{0}-y^{0}\right)\left\langle X_{S}\left|\frac{1}{\mathcal{P}_{\perp}^{2}} O_{S}^{A}(\hat{x}) \frac{1}{\mathcal{P}_{\perp}^{2}} O_{S}^{B}(\hat{y}) \rho_{B}\right| X_{S}\right\rangle\left\langle\tilde{X}_{n}\left|O_{n}^{A}(x) O_{n}^{B}(y)\right| Y_{1}\right\rangle \\
& \left\langle Y_{1} \bar{X}_{n}\left|\chi_{n}(0) \frac{\not h}{2}\right| 0\right\rangle\left\langle 0\left|\bar{\chi}_{n}(0) \delta^{2}\left(\mathcal{P}_{\perp}\right) \delta^{2}\left(Q-\mathcal{P}^{-}\right) \mathcal{M}_{V}\right| \tilde{X}_{n} \bar{X}_{n}\right\rangle
\end{aligned}
$$

We can now write $\Theta(t)=1 / 2(1+\operatorname{sgn}(t))$ separating out the terms corresponding to Unitary evolution from the dissipative.

$$
\mathcal{G}=\mathcal{G}_{U}+\mathcal{G}_{D}
$$

The piece corresponding to unitary evolution reads

$$
\begin{aligned}
\mathcal{G}_{U}= & \int d^{4} x \int d^{4} y \operatorname{sgn}\left(x^{0}-y^{0}\right)\left\langle X_{S}\left|\frac{1}{\mathcal{P}_{\perp}^{2}} O_{S}^{A}(\hat{x}) \frac{1}{\mathcal{P}_{\perp}^{2}} O_{S}^{B}(\hat{y}) \rho_{B}\right| X_{S}\right\rangle\left\langle\tilde{X}_{n}\left|O_{n}^{A}(x) O_{n}^{B}(y)\right| Y_{1}\right\rangle \\
& \left\langle Y_{1} \bar{X}_{n}\left|\chi_{n}(0) \frac{\not h}{2}\right| 0\right\rangle\left\langle 0\left|\bar{\chi}_{n}(0) \delta^{2}\left(\mathcal{P}_{\perp}\right) \delta^{2}\left(Q-\mathcal{P}^{-}\right) \mathcal{M}_{V}\right| \tilde{X}_{n} \bar{X}_{n}\right\rangle
\end{aligned}
$$

This piece cancels out with the corresponding Unitary evolution term from double Glauber insertions on the other side of the cut (By interchanging the variables $p_{Y_{1}}$ and $p_{\tilde{X}_{n}}$ ). The Dissipative piece, on the other hand no longer contains any time ordering. Then, as before in eq. (3.47), using the translational invariance of the QGP medium,

$$
\left\langle X_{S}\left|\frac{1}{\mathcal{P}_{\perp}^{2}} O_{S}^{A}\left(x_{\perp}, x^{-}\right) \frac{1}{\mathcal{P}_{\perp}^{2}} O_{S}^{B}\left(y_{\perp}, y^{-}\right) \rho_{B}\right| X_{S}\right\rangle=\int \frac{d^{4} k}{(2 \pi)^{4} k_{\perp}^{4}} e^{i(\hat{x}-\hat{y}) \cdot k} D_{>}^{A B}(k)
$$

The factorization formula now becomes

$$
\begin{aligned}
\Sigma_{V}^{(2)}= & -\frac{1}{2} V \times\left|C_{G}(Q)\right|^{2} H(Q, \mu) \int d^{2} \vec{q}_{J S} S\left(\vec{q}_{J S}\right) \int d^{2} \vec{q}_{\bar{n}} \mathcal{J}_{\bar{n}}^{\perp}\left(e_{\bar{n}}, \vec{q}_{\bar{n}}\right) \\
& \int \frac{d^{4} k}{(2 \pi)^{4} k_{\perp}^{4}} D_{>}^{A B}(k) \int d^{2} \vec{q}_{n} \int d^{4} x \int d^{4} y e^{i(\hat{x}-\hat{y}) \cdot k} J_{n}^{A B}\left(e_{n}, \vec{q}_{n}, x, y\right) \\
& \times \delta^{2}\left(\vec{q}_{T}+\vec{q}_{J S}+\vec{q}_{n}+\vec{q}_{\bar{n}}\right)
\end{aligned}
$$


with

$$
J_{n}^{A B}\left(e_{n}, \vec{q}_{n}, x, y\right)=\left\langle\tilde{X}_{n}\left|O_{n}^{A}(x) O_{n}^{B}(y)\right| Y_{1}\right\rangle\left\langle Y_{1} \bar{X}_{n}\left|\chi_{n}(0) \frac{\not h}{2}\right| 0\right\rangle\left\langle 0\left|\bar{\chi}_{n}(0) \delta^{2}\left(\mathcal{P}_{\perp}\right) \delta^{2}\left(Q-\mathcal{P}^{-}\right) \mathcal{M}_{V}\right| \tilde{X}_{n} \bar{X}_{n}\right\rangle
$$

We can now insert a complete set of states and perform the integrals over $x, y$, This will enable us to eliminate the phase space integrals $p_{Y 1}$ and $\tilde{X}_{n}$, resulting in a factor of $\mathrm{t}$.

$$
\begin{aligned}
& \int d^{4} x d^{4} y e^{-i k \cdot(\hat{x}-\hat{y})} J_{n}^{A B}\left(e_{n}, \vec{q}_{n} x, y\right) \\
& =\frac{t}{\left[p_{\tilde{Y}}^{-}\right]^{2}}\left\langle p_{\tilde{Y}}+k\left|O_{n}^{A}(0)\right| \tilde{Y}_{n}\right\rangle\left\langle\tilde{Y}_{n}\left|O_{n}^{B}(0)\right| p_{\tilde{Y}}+k\right\rangle\left\langle p_{\tilde{Y}}+k, \bar{X}_{n}\left|\chi_{n}(0) \frac{\not h}{2}\right| 0\right\rangle \\
& \left\langle 0\left|\bar{\chi}_{n}(0) \delta^{2}\left(\mathcal{P}_{\perp}\right) \delta^{2}\left(Q-\mathcal{P}^{-}\right) \mathcal{M}_{V}\right| p_{\tilde{Y}}+k, \bar{X}_{n}\right\rangle
\end{aligned}
$$

We can factor out the vacuum cross section as before and write,

$$
\Sigma_{V}^{(2)}=-\frac{1}{2} t \times\left|C_{G}(Q)\right|^{2} \int d^{2} b e^{i \vec{b} \cdot \vec{q}_{T}} \Sigma^{(0)}\left(\vec{b}, e_{n}, e_{\bar{n}}\right) \int \frac{d^{2} k_{\perp}}{(2 \pi)^{4}} S_{G}^{A B}\left(k_{\perp}\right) \mathcal{J}_{n, V}^{A B}\left(e_{n}, b, \vec{k}_{\perp}\right)
$$

where the Soft function $S_{G}^{A B}$ is identical to the one for $\Sigma_{R}^{(2)}$ defined in eq. (3.51) and

$$
\begin{aligned}
\mathcal{J}_{n, V}^{A B}(b)= & \frac{1}{k_{\perp}^{2} \mathcal{J}_{n}^{\perp}\left(e_{n}, b\right)} \frac{1}{\left[p_{\tilde{Y}}^{-}\right]^{2}} \int \frac{d^{2} q_{n} e^{-i \vec{q}_{n} \cdot \vec{b}}}{(2 \pi)^{2}}\left\langle p_{\tilde{Y}}+k\left|O_{n}^{A}(0)\right| \tilde{Y}_{n}\right\rangle\left\langle\tilde{Y}_{n}\left|O_{n}^{B}(0)\right| p_{\tilde{Y}}+k\right\rangle \\
& \left\langle p_{\tilde{Y}}+k, \bar{X}_{n}\left|\chi_{n}(0) \frac{\hbar}{2}\right| 0\right\rangle\left\langle 0\left|\bar{\chi}_{n}(0) \delta^{2}\left(\mathcal{P}_{\perp}\right) \delta^{2}\left(Q-\mathcal{P}^{-}\right) \mathcal{M}_{V}\right| p_{\tilde{Y}}+k, \bar{X}_{n}\right\rangle
\end{aligned}
$$

We can now combine the two simplified terms to write the final form of our factorization formula

$$
\Sigma_{R}^{(2)}+\left\{\Sigma_{V}^{(2)}+c . c\right\}=t \times\left|C_{G}(Q)\right|^{2} \Sigma^{(0)}\left(\vec{q}_{T}, e_{n}, e_{\bar{n}}\right) \otimes_{q_{T}} \int d^{2} k_{\perp} S_{G}^{A B}\left(k_{\perp}\right) \mathcal{J}_{n, \mathrm{Med}}^{A B}\left(e_{n}, \vec{q}_{T}, k_{\perp}\right)
$$

with an effective medium induced jet function

$$
\mathcal{J}_{n, \mathrm{Med}}^{A B}\left(e_{n}, \vec{q}_{T}\right)=\mathcal{J}_{n, R}^{A B}\left(e_{n}, \vec{q}_{T}, \vec{k}_{\perp}\right)-\mathcal{J}_{n, V}^{A B}\left(e_{n}, \vec{q}_{T}, \vec{k}_{\perp}\right)
$$

in terms of $\mathcal{J}_{n, R}^{A B}$ and $\mathcal{J}_{n, V}^{A B}$ defined in eq. (4.12) and eq. (4.24).

In the next section, we will consider the one loop corrections for the functions in this factorization formula.

\section{One loop results for medium induced functions}

The factorization formula at next to leading order in the Glauber operator expansion reveals two new functions beyond those already present in the vacuum result: a medium Soft function and a medium induced jet function. Of these, the medium soft function $S_{G}^{A B}$ defined in eq. (3.51) contains all the information about the structure of the medium and is independent of the observable defined on the final state jet. Hence, it can be thought of as a universal structure function for the medium. An explicit computation of this function 
now requires us to assume some form for the medium density matrix. A relevant choice is a medium in thermal equilibrium at temperature $T \ll Q$ where the EFT applies for which

$$
\rho_{B}=\frac{e^{-\beta H_{s}}}{\operatorname{Tr}\left[e^{-\beta H_{s}}\right]}
$$

where $H_{s}$ is the Soft sector Hamiltonian which is identical to the full QCD Hamiltonian.

The medium induced jet function encodes the modification of the jet due to the interaction with the medium. The complete set of corrections for this function at one loop involves:

- Elastic collisions of the jet partons with the medium.

- Medium induced radiation.

In this paper, we will only consider the one loop corrections for the hard vertex, which correspond to the corrections from elastic collisions of the vacuum evolved jet partons with the medium. The corrections to the Glauber vertex which corresponds to medium induced radiation are presented elsewhere [92]. Since the medium Soft function is a correlator of the Galuber Soft operator, we therefore compute the Soft function to tree level. With the assumption of a thermal medium, the tree level soft function is then simply the Wightman function in the thermal bath. This was already computed in [84]. We present the result here for convenience.

$$
\begin{aligned}
S_{G}^{A B,(0)}\left(\vec{k}_{\perp}\right)= & \frac{\delta^{A B}}{2 k_{\perp}^{2}} \int \frac{d^{2} p_{\perp}}{(2 \pi)^{3}} \int_{0}^{\infty} d p^{+}\left\{n_{F}\left(\left|\frac{p^{+}}{2}+\frac{p_{\perp}^{2}}{2}\right|\right)\left[1-n_{F}\left(\left|\frac{p^{+}}{2}+\frac{\left(\vec{p}_{\perp}+\vec{k}_{\perp}\right)^{2}}{2 p^{+}}\right|\right)\right]\right. \\
& \left.+n_{F}\left(\left|\frac{p^{+}}{2}+\frac{\left(\vec{p}_{\perp}+\vec{k}_{\perp}\right)^{2}}{2 p^{+}}\right|\right)\left[1-n_{F}\left(\left|\frac{p^{+}}{2}+\frac{p_{\perp}^{2}}{2}\right|\right)\right]\right\}
\end{aligned}
$$

This result is written in terms of the Fermi distribution function assuming that the thermal bath has fermions. A complete calculation would also include a similar term with Bose distribution functions to account for gluons in the thermal bath, but for simplicity we will not consider those terms till we do a phenomenological analysis.

We can now look at the medium jet function, specifically considering the one loop corrections from elastic scattering. At tree level we have in impact parameter space

$$
\mathcal{J}_{n, \text { Med }}^{A B,(0)}\left(\vec{k}_{\perp}, b, e_{n}\right)=\frac{e^{-i \vec{k}_{\perp} \cdot \vec{b}}-1}{k_{\perp}^{2}}
$$

The details of the one loop computation for the elastic collisions are computed in appendix B.

$$
\mathcal{J}_{n, \text { Med }}^{A B(1)}=\frac{\frac{1}{2} \delta^{A B}}{k_{\perp}^{2}}(Q(b)+G(b))
$$


where the result is written in terms of Quark and Gluon Glauber operator contributions

$$
\begin{aligned}
Q(b)= & \frac{\alpha_{s} C_{F} e^{-i \overrightarrow{k_{\perp}} \cdot \vec{b}}}{2 \pi}\left[\int_{z_{c}}^{1-z_{c}} d z p_{g q}(z)\left\{\Theta\left(z_{c}-\frac{y}{e_{n}+y}\right) \ln \left(\frac{-B(z)}{e_{n}(1-z)}\right)-\Theta\left(z_{c}-\frac{e_{n}}{e_{n}+y}\right) \ln \frac{B(z) e_{n}}{y M}\right\}\right. \\
& \left.+\Theta\left(\frac{e_{n}}{e_{n}+y}-z_{c}\right) \Theta\left(\frac{y}{e_{n}+y}-z_{c}\right)\left\{\int_{z_{c}}^{\frac{e_{n}}{e_{n}+y}} d z p_{g q}(z) \ln \left(\frac{-B(z)}{e_{n}(1-z)}\right)-\int_{\frac{e_{n}}{e_{n}+y}}^{1-z_{c}} d z p_{g q}(z) \ln \frac{B(z) e_{n}}{y M}\right\}\right] \\
& +\frac{\alpha_{s} C_{F}}{2 \pi}\left(e^{-i \vec{k}_{\perp} \cdot \vec{b}}-1\right) \int_{1-z_{c}}^{1} p_{g q}(z) \ln \frac{m_{D}^{2} b^{2} e^{2 \gamma_{E}}(1-z)}{4}
\end{aligned}
$$

The Gluon operator contribution $\mathrm{G}(\mathrm{b})$ is given by the difference $R_{g}-V_{g}$, where

$$
\begin{aligned}
R_{g}= & \frac{\alpha_{s} N_{c} e^{-i \vec{k}_{\perp} \cdot \vec{b}}}{2 \pi}\left[\int_{z_{c}}^{1-z_{c}} d z p_{g q}(z)\left\{\Theta\left(z_{c}-\frac{y}{e_{n}+y}\right) \ln \frac{A(z)}{M}-\Theta\left(z_{c}-\frac{e_{n}}{e_{n}+y}\right) \ln \left(\frac{-A(z)}{y(1-z)}\right)\right\}\right. \\
& \left.-\Theta\left(\frac{e_{n}}{e_{n}+y}-z_{c}\right) \Theta\left(\frac{y}{e_{n}+y}-z_{c}\right)\left\{\int_{z_{c}}^{\frac{y}{e_{n}+y}} d z p_{g q}(z) \ln \left(\frac{-A(z)}{y(1-z)}\right)-\int_{\frac{y}{e_{n}+y}}^{1-z_{c}} d z p_{g q}(z) \ln \frac{A(z)}{M}\right\}\right] \\
& -\frac{\alpha_{s} N_{c}}{2 \pi} e^{-i \vec{k}_{\perp} \cdot \vec{b}} \int_{1-z_{c}}^{1} p_{g q}(z) \ln \frac{m_{D}^{2} b^{2} e^{2 \gamma_{E}}(1-z)}{4}
\end{aligned}
$$

written in terms of

$p_{g q}(z)=\frac{1+(1-z)^{2}}{z}, \quad y=\frac{4 k_{\perp}^{2}}{Q^{2}}, \quad M=\frac{4 m_{D}^{2}}{Q^{2}}, \quad A(z)=e_{n} z-(1-z) y, \quad B(z)=z y-e_{n}(1-z)$

and $V_{g}$ is obtained by simply evaluating $R_{g}$ ay $k_{\perp}=0$. The integrals over z can be done exactly analytically but are not too illuminating. The contributions $\mathrm{Q}(\mathrm{b})$ and $\mathrm{G}(\mathrm{b})$ go to 0 as $k_{\perp}$ goes to 0 so that it is a purely medium induced result. There are two effects to note here

- These radiative corrections result from purely elastic collisions of the jet with the medium and do not include any medium induced Bremsstrahlung, which will be accounted for in a companion paper [92]. The result does not have any UV or rapidity divergences and hence does not induce any anomalous dimension for the medium jet function. Therefore, there is no resummation to be done for elastic collision contributions.

- The result is sensitive to the IR cut-off $m_{D}$ in the form of a logarithm of $\sim \ln e_{n} / M$, $\ln b m_{D}$. This appears due to the IR pole when the gluon becomes collinear to the quark. For a purely vacuum background, the collinear pole cancels between real and virtual diagrams for an IRC (Infra Red Collinear) safe observable like the jet mass. However, incoherent elastic collision of the jet parton with the medium can transfer enough transverse momentum which can remove this pole from the real diagram if the resulting jet mass is greater than the imposed values of $e_{n}$. This leaves the contribution from the virtual diagram uncanceled resulting in the sensitivity to $m_{D}$. Similarly for the $q_{T}$ measurement, the collinear pole shifts away from $q_{T}=0$ due to the gain of additional $k_{T}$ from the medium resulting in non-cancellation. A possible resummation of these logarithms would require us to separate the scale $e_{n}, q_{T}$ from $M^{2}$, which will require a further matching to the EFT at the scale $m_{D}$. This is beyond the scope of this paper. 


\section{The master equation}

We can now gather all the pieces and write down the Master equation for the density matrix evolution. Since the radiative corrections to the medium induced corrections that we have considered in the previous section do not lead to any new anomalous dimensions, we simply include these corrections as a fixed order correction on top of a resummed vacuum cross section (appendix A). Combining all the pieces from the previous section and using them in eq. (3.21), we can write

$$
\begin{aligned}
& \operatorname{Tr}[\rho(t) \mathcal{M}] \equiv \Sigma\left(e_{n}, \vec{q}_{T}, t\right) \\
& =\left[\Sigma_{\text {Resum }}^{(0)}\left(e_{n}, \vec{q}_{T}\right)+t\left|C_{G}(Q)\right|^{2} \Sigma_{\text {Resum }}^{(0)}\left(e_{n}, \vec{q}_{T}\right) \otimes_{q_{T}} \int d^{2} k_{\perp} S_{G}^{A B}\left(k_{\perp}\right) \mathcal{J}_{n, \text { Med }}^{A B}\right]+O\left(H_{G}^{3}\right)+\ldots
\end{aligned}
$$

We can write the evolution equation in a suggestive form by going to impact parameter space $(\vec{b})$ which is conjugate to $\vec{q}_{T}$,

$$
\Sigma(t, \vec{b})=\Sigma^{(0)}(\vec{b})\left[1+\frac{t}{\lambda_{\operatorname{mfp}}(\vec{b})}\right]
$$

where

$$
\lambda_{\mathrm{mfp}}^{-1}(b)=\left|C_{G}\right|^{2} \int d^{2} k_{\perp} S_{G}^{A B}\left(k_{\perp}\right) \mathcal{J}_{n, \text { Med }}^{A B}(\vec{b})
$$

is an emergent energy scale that can be thought of as the inverse mean free path of the jet in the medium. $t$ is the time of propagation of the jet in the medium. If the medium is very dilute then the mean free path (mfp) can be large compared to $t$ in which case eq. (6.1) is a good enough approximation. On the other hand, for the case of a denser medium we can have $t \sim \lambda_{\mathrm{mfp}}$, in which case it becomes necessary to resum the series in $t / \lambda_{\mathrm{mfp}}$ to all orders.

We first relate the trace over the density matrix to the scattering cross section, noting that

$$
\frac{d \sigma\left(e_{n}\right)}{d^{2} \vec{q}_{T}}(t)=\mathcal{N} \frac{\operatorname{Tr}[\rho(t) \mathcal{M}]}{V}
$$

where $\mathcal{N}$ is a normalization factor that depends on the initial state kinematics which we can absorb in the born level cross section.

$$
\frac{d \sigma\left(e_{n}, e_{\bar{n}}, t\right)}{d^{2} \vec{q}_{T}}=\left.\frac{d \sigma\left(e_{n}, e_{\bar{n}}\right)}{d^{2} \vec{q}_{T}}\right|_{\text {Resum }} ^{\text {Vac }}+\left.t\left|C_{G}(Q)\right|^{2} \frac{d \sigma\left(e_{n}, e_{\bar{n}}\right)}{d^{2} \vec{q}_{T}}\right|_{\text {Resum }} ^{\text {Vac }} \otimes_{q_{T}} \int d^{2} k_{\perp} S_{G}^{A B}\left(k_{\perp}\right) \mathcal{J}_{n, \text { Med }}^{A B}
$$

Throughout this paper, we have worked in the regime where the medium lifetime t and the mean free path $\lambda_{\mathrm{mfp}}$ are much longer than the formation time $t_{F}$ of the jet partons and the relaxation time in the QGP bath $1 / T$. This means that after each interaction with the medium, the jet partons go on-shell before the next interaction with the medium. At the same time, the medium loses any memory of the jet between successive interactions. The hierarchy of scales also implies that there is no quantum interference between successive interactions of the jet with the medium so that the LPM effect is not relevant here. Therefore, the evolution of the jet at any time depends entirely on the state of the jet partons at that time, making this a Markovian process. We can therefore consider a evolution time step 
$t-0=\Delta t \gg 1 / T, t_{F}$ and at the same time $\Delta t \ll \lambda_{\mathrm{mfp}}$. This allows us to take the limit $t \rightarrow \infty$ while talking about a single interaction of the jet with the medium, thus imposing momentum conservation, while at the same time taking $t \rightarrow 0$ while talking about multiple jet medium interactions.

We can therefore write an evolution equation for our observable as a function of the time of propagation in the QGP medium.

$$
\partial_{t} \frac{d \sigma\left(e_{n}, e_{\bar{n}}, t\right)}{d^{2} \vec{q}_{T}}=\left|C_{G}(Q)\right|^{2} \frac{d \sigma\left(e_{n}, e_{\bar{n}}, t\right)}{d^{2} \vec{q}_{T}} \otimes_{q_{T}} \int d^{2} k_{\perp} S_{G}^{A B}\left(k_{\perp}\right) \mathcal{J}_{n, \text { Med }}^{A B}\left(e_{n}, \vec{q}_{T}, \vec{k}_{\perp}\right)
$$

The solution for this equation will resum the leading " $t$ " enhanced terms for all higher power contributions of $H^{G}$. The physical picture we have is a summation of multiple incoherent interactions of the jet with the medium. This can now be solved by going to impact parameter space. This yields the result

$$
\frac{d \sigma\left(e_{n}, e_{\bar{n}}, t\right)}{d^{2} \vec{q}_{T}}=\int d^{2} \vec{b} e^{i \vec{b} \cdot \vec{q}_{T}} \sigma_{\text {resum }}^{\mathrm{vac}}\left(e_{n}, e_{\bar{n}}, \vec{b}\right) e^{t\left|C_{G}(Q)\right|^{2} \int d^{2} k_{\perp} S_{G}^{A B}\left(k_{\perp}\right) \mathcal{J}_{n, \mathrm{Med}}^{A B}\left(e_{n}, \vec{b}, \vec{k}_{\perp}\right)}
$$

where $\sigma^{\text {vac }}\left(e_{n}, e_{\bar{n}}, \vec{b}\right)$ is just the inverse Fourier transform for the resummed vacuum cross section.

$$
\sigma_{\text {resum }}^{\mathrm{vac}}\left(e_{n}, e_{\bar{n}}, \vec{b}\right)=\left.\int \frac{d^{2} \vec{q}_{T} e^{-i \vec{q}_{T} \cdot \vec{b}}}{(2 \pi)^{2}} \frac{d \sigma\left(e_{n}, e_{\bar{n}}\right)}{d^{2} \vec{q}_{T}}\right|_{\text {Resum }} ^{\mathrm{Vac}}
$$

Eq. (6.5) is the main result of this paper. While the result gives us a closed form expression, we still need to do the integrals numerically from this point onwards. A realistic comparison with data will need us to include the effects of nuclear/ hadronic pdfs and we leave a detailed phenomenological study based on this framework for the future. At the same time, the corrections to the Glauber vertex, which we have not included in this paper will likely induce new UV and rapidity divergences in our medium soft and jet functions whose computation and resummation is considered in [92].

\section{Comparison with other formalisms}

The most direct comparsion of the formalism introduced in this paper can be made with the earlier attempt to use SCET for heavy ion collisions [53-57]. This $\mathrm{SCET}_{G}$ approach treats the interaction of the jet with the medium in terms of a background Glauber gauge field, integrating out the propagating degrees of freedom of the medium. The framework also employs an expansion in the interaction with the medium, called the opacity expansion, which is similar to the expansion in the Glauber Hamiltonian made in this paper. The main difference is the treatment of soft physics, which is altogether absent in the $\mathrm{SCET}_{G}$ approach since the soft medium degrees of freedom at the scale of the temperature and the radiation that appears at this scale are not incorporated. Only collinear physics is included and this is insufficient to establish gauge invariance or consistency of factorization. In particular, as is shown in [92], which is a companion paper to the formalism introduced here, the radiative corrections for the medium structure function which captures the soft physics in the medium lead to UV and rapidity divergences which are absent in the $\mathrm{SCET}_{G}$ framework 
since the medium is treated as a classical background field. The corresponding rapidity renormalization group is just the BFKL equation, which is a new result in Heavy Ion physics that has not been captured by any previous formalism. The rapidity divergence is then canceled by the collinear jet function, while the UV divergences are canceled by the Hard function thus establishing renormalization scale independence of the cross section. This also induces large logarithms which can then be resummed by solving the renormalization group equations which are also absent in earlier formalisms. It is to be noted that, the inclusion of soft physics of the medium is not dependent on the details of jet observable but is part of the medium structure function defined in eq. (3.46) which can be thought of as the Parton Distribution function of the medium. The other advantage of having a factorization formula, which is not available so far in literature is that it allows for a systematic way to improve the accuracy of the calculation simply by computing each factorized function (whose operator definitions are known) to better accuracy. There are other formalisms in literature such as BDMPS-Z [4, 6], AMY [14], deal with multiple coherent interactions of the jet with the medium which is true when the mean free path of the jet in the medium is comparable to its coherence length leading to interference between successive interactions with the medium (LPM effect). The case considered in this paper is that of a long lived dilute medium where such interference effects are not important. This precludes a direct comparison with the formalisms above until a future work where the framework here can be extended to a dense medium. However, none of these frameworks include soft physics at the scale of the temperature. which appears to be an important piece of the full story. We can still say a lot about what the structure of the factorization and radiative corrections in our approach should look like for these cases, which is discussed at the end of the next section. We can also compare the general approach to the problem highlighting why an EFT can be considered advantageous over other formalisms

- It provides an explicit separation of physics at widely separated scales in the form of a factorization formula for the jet observables.

- It gives a gauge invariant operator definition for the factorized functions.

- The factorization does not rely on perturbation theory but only on a separation of scales. So the factorization holds even when some of the functions become nonperturbative. Thus, it gives a clear boundary between the perturbatively calculable and non-calculable physics. This is in exact analogy with factorization formulas for ep and pp experiments such as Deep Inelastic scattering (DIS) and Drell-Yan where the universal physics of the proton is defined by a Parton Distribution Function (PDF). This has the advantage that if some of the factorized functions become non-perturbative, there is a possibility of computing them from lattice/quantum computers.

- Lastly, the separation of scales allows us to resum large logarithms in the ratio of these scales via Renormalization group equation for the factorized functions. This is systematically improvable order by order in Logarithmic accuracy and has already led to precise predictions in pp and ep experiments. 


\section{Summary and outlook}

In this paper, I develop an Effective Field Theory (EFT) framework to compute jet substructure observables for heavy ion collision experiments. I consider dijet events that happen at the periphery of the collision so that one of the jets evolves through vacuum while the other travels through the Quark Gluon Plasma medium that is created in the background. The jets are groomed using a grooming algorithm in order to mitigate effects of soft contamination from Multi-parton interactions as well as the QGP medium. This means that the final state measurements do not include any soft hadrons from the cooling QGP medium. We can then only follow the evolution of the reduced density matrix of the jet tracing over the QGP bath. This effectively treats the jet as an open quantum system interacting with a thermal bath and allows us to derive a Lindblad type master equation for the density matrix evolution.

I measure two quantities on the final state di-jet configuration: the transverse momentum imbalance between the jets as well as a jet mass constraint imposed on each jet which restricts the radiation inside the large radius jets to a collinear core. This automatically ensures that we select events where the radiation is not sensitive to the edge of the jet. This translates to an insensitivity to jet selection bias and allows a direct comparison of jet substructure modification for the same hard events in pp and HIC.

I work in the simple case where the length of the medium and the mean free path of the jet are much longer than all other time scales, particularly the jet formation time $t_{F}$, which is the time scale over which jet partons maintain coherence, and the relaxation time in the bath $1 / \mathrm{T}$. As a result, the dominant contribution to the evolution of the jet in the medium comes from the regime where the partons created in the hard interaction and subsequent vacuum shower go on-shell before they interact with the medium. This happens due to the lack of quantum coherence between the hard and medium interactions. I derive a factorization formula for the observable assuming a forward scattering of the jet. This allows us to cleanly separate out the physics at different scales in terms of manifestly gauge invariant operators. The factorization holds independent of the exact form of the medium density matrix, with the only qualification that the medium be translationally invariant. As explained after eq. (3.46), this is not a necessary condition for factorization and can be relaxed. The final formula is derived in terms of the vacuum cross section and a medium soft and jet function. The factorization formula will serve as a template for any jet substructure observable that we may wish to compute and reveals certain universal features.

- The physics of the medium is completely captured by a medium Soft function which is a correlator of Soft scaling fields in the background of the medium density matrix. This can be thought of as a "Medium Structure Function" (MSF) which is analogous to the Parton Distribution function (PDF) which encodes the longitudinal structure of hadrons. In this paper, I have only computed this to tree level in a thermal background. The radiative corrections have been considered in a companion paper [92] which lead to both UV and rapidity anomalous dimensions for this function. In particular, they lead to a BFKL evolution equation for the medium structure function. 
- The modification of the jet due to its interaction with the medium is captured by the medium jet function. In this paper, we have only considered the corrections from elastic collisions of the jet partons with the medium. This leads to UV finite corrections, which are however sensitive to medium IR cut-off scale $m_{D}$. In a high temperature-weak coupling regime, $m_{D} \sim g T$ is hierarchically separated from the scale of the observable and leads to large logarithms. These can be resummed by doing a further matching of the jet function to an EFT at scale $m_{D}$, which we leave for the future. The other radiative corrections arise due to medium induced Bremsstrahlung have been computed in another paper [92].

The jet-medium interaction leads to an emergent time scale $\lambda_{\mathrm{mfp}}$ which is the mean free path of the jet in the medium. If the medium size is comparable to or larger than this length scale, then it becomes necessary to resum multiple incoherent interactions of the jet with the medium. This is achieved by solving a Lindblad type evolution equation which can be solved analytically in terms of a resummed vacuum cross section and medium induced functions.

The assumption of a long lived medium which renders all interactions of the jet incoherent is an idealization but is an important step towards addressing a realistic scenario. In particular, we would like to consider the following phenomenologically relevant cases of increasing complexity

1. The medium exists for a short time comparable to or shorter than the formation time of the jet but the medium is dilute. This means that the mean free path is large compared to the medium size and only a single interaction of the jet with the medium is relevant. In this case the jet partons wont go on-shell before they interact with the medium. This would mean a hard interaction that creates the jet and the forward scattering from the medium can have interference diagrams. Since the formation time for Soft radiation is small, we conjecture that the medium structure function remains unchanged and only the jet function will get modified. This case of a dilute short lived medium is currently being investigated [91].

2. A more complicated case would be that the mean free path can be comparable to or smaller than the formation time of the jet which in turn is comparable to the size of the medium. This would mean that apart from hard-medium interaction interference, multiple interactions of the jet with the medium are important and there is quantum interference between successive interactions (LPM effect). Again, this will only impact the jet function. This entails defining and computing jet functions at higher orders in the Glauber Hamiltonian and summing the series along with all the radiative corrections at each order. This case of a dense short lived medium will be considered in a future work.

While I have treated the initial hard interaction as an $e^{+} e^{-}$collision for ease of analysis, this can be easily extended to the realistic case of hadronic collisions which will also require us to input nuclear pdfs for comparison with data. The factorization for the case pf pp collisions is more involved and is written in terms of the so called Beam function [93] which includes the 
effects of initial state radiation. So we will get a contribution to our perturbative transverse momentum observable $\vec{q}_{T}$ from the initial state radiation. For $\vec{q}_{T} \gg \Lambda_{Q C D}$, this beam function can be matched to the usual parton distribution functions. As a concrete example, the authors in [94] extended the factorization for the jet observable called angularities for the case of dijet production in pp collisions which is closely related to the jet mass measurement we have imposed in this paper. We leave the detailed phenomenological analysis based on this framework for future work.

\section{Acknowledgments}

I thank Xiaojun Yao and Krishna Rajagopal for useful discussions on several conceptual aspects. This work is supported by the Office of Nuclear Physics of the U.S. Department of Energy under Contract DE-SC0011090 and Department of Physics, Massachusetts Institute of Technology.

\section{A Operator definitions and one loop results for vacuum evolution}

In this appendix we give the operator definitions of the factorization elements that appear in the vacuum and their NLO expansions. From those we determine the renormalization group equations, and corresponding anomalous dimensions. Part of these results are already available in literature. The jet function is the one we compute here while quoting results for other functions.

\section{A.1 Hard function}

The one loop hard function for the process $e^{+} e^{-} \rightarrow q \bar{q}$ is $([95,96])$

$$
H(\mu)=1+\frac{\alpha_{s} C_{F}}{2 \pi}\left(-\ln ^{2} \frac{\mu^{2}}{Q^{2}}-3 \ln \frac{\mu^{2}}{Q^{2}}-8+\frac{7}{6} \pi^{2}\right)
$$

which yields the anomalous dimension

$$
\gamma_{\mu}^{H}=-2 \frac{\alpha_{s} C_{F}}{\pi} \ln \frac{\mu^{2}}{Q^{2}}-3 \frac{\alpha_{s} C_{F}}{\pi}
$$

\section{A.2 Jet function}

The vacuum jet function with a cumulative jet mass measurement along with transverse momentum imbalance is given by

$$
\mathcal{J}_{n}^{\perp}\left(\vec{q}_{T}, e_{n}\right)=\frac{(2 \pi)^{3}}{N_{c}} \operatorname{tr}\left\langle 0\left|\frac{\hbar}{2} \chi_{n} \delta\left(Q-\mathcal{P}^{-}\right) \delta^{2}\left(\mathcal{P}^{\perp}\right) \delta^{2}\left(\vec{q}_{T}-\mathcal{P}_{X_{n} \notin \mathrm{gr}}^{\perp}\right) \Theta\left(e_{n}-\mathcal{E}_{X_{n} \in \mathrm{gr}}\right)\right| X_{n}\right\rangle\left\langle X_{n}\left|\bar{\chi}_{n}(0)\right| 0\right\rangle
$$

This defines the vacuum evolved $\mathrm{n}$ jet function and we have a similar definition for the vacuum evolved $\bar{n}$ jet function. The Renormalization group equations for each of these vacuum jet functions is identical and hence we will only present the result for the $\mathrm{n}$ jet function. We will work in impact parameter space and give results for

$$
\mathcal{J}_{n}^{\perp}\left(\vec{b}, e_{n}\right)=\int \frac{d^{2} \vec{q}_{T}}{(2 \pi)^{2}} e^{-i \vec{q}_{T} \cdot \vec{b}} \mathcal{J}_{n}^{\perp}\left(\vec{q}_{T}, e_{n}\right)
$$




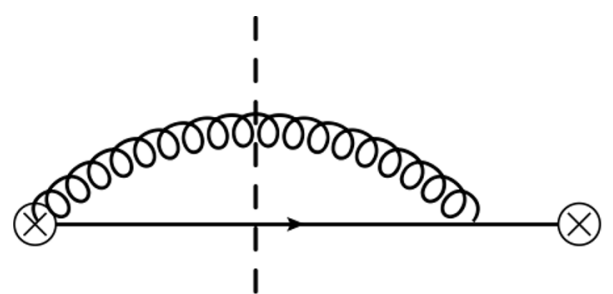

(a)

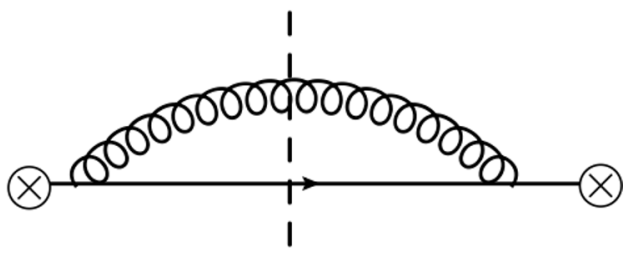

(b)

Figure 2. Real emission diagrams for vacuum jet function at the amplitude square level. The dashed line indicates the cut. Diagram (a) has a real gluon emission from the collinear Wilson line attached to the hard vertex which creates the initiating n quark, denoted by $\otimes$ along with a single insertion from $H_{n \text {,int }}$ which describes interactions between n collinear partons. Diagram (b) comes from two insertions of $H_{n \text {,int }}$. Since this is vacuum evolution there are no Glauber exchanges with the medium here.

At tree level,

$$
\mathcal{J}_{n}^{\perp(0)}=\frac{1}{(2 \pi)^{2}}
$$

At one loop we have two real and two virtual diagrams. Since we know that the vacuum cross section is an IR finite quantity, we can directly use dim reg for regulating both UV and IR divergences. In that case, the virtual diagrams are scaleless and evaluate to 0 in dim. reg. This function obeys two RG equations

$$
\begin{aligned}
\mu \frac{d}{d \mu} \mathcal{J}_{n}^{\perp}\left(\vec{b}, e_{n}, \mu, \nu\right) & =\gamma_{\mu}^{J} \mathcal{J}_{n}^{\perp}\left(\vec{b}, e_{n}, \mu, \nu\right) \\
\mu \frac{d}{d \nu} \mathcal{J}_{n}^{\perp}\left(\vec{b}, e_{n}, \mu, \nu\right) & =\gamma_{\nu}^{J} \mathcal{J}_{n}^{\perp}\left(\vec{b}, e_{n}, \mu, \nu\right)
\end{aligned}
$$

The two real diagrams are shown in figure 2 .

$$
\begin{aligned}
R_{a}= & 4 g^{2} C_{F} \mu^{2 \epsilon} \nu^{\eta} \int \frac{d^{d} q}{(2 \pi)^{d-1}} \delta\left(q^{2}\right) \frac{Q\left|q^{-}\right|^{-\eta}}{q^{-} Q\left(q^{+}+\frac{q_{\perp}^{2}}{Q-q^{-}}\right)} \mathcal{M}_{J} \\
R_{b}= & 2 g^{2} C_{F} \mu^{2 \epsilon} \int \frac{d^{d} q}{(2 \pi)^{d-1}} \delta\left(q^{2}\right) \frac{q_{\perp}^{2}}{q^{-}\left(Q-q^{-}\right)^{2}\left(q^{+}+\frac{q_{\perp}^{2}}{Q-q^{-}}\right)^{2}} \mathcal{M}_{J} \\
\mathcal{M}_{J}= & \left\{\Theta\left(q^{-}-Q z_{c}\right) \Theta\left(\left(Q-q^{-}\right)-Q z_{c}\right) \Theta\left(E_{J}^{2} e_{n}-Q\left(q^{+}+\frac{q_{\perp}^{2}}{Q-q^{-}}\right)\right)\right. \\
& \left.\quad+\delta^{2}\left(\vec{q}_{T}-\vec{q}_{\perp}\right) \Theta\left(\left(Q-q^{-}\right)-Q z_{c}\right) \Theta\left(Q z_{c}-q^{-}\right)+\delta^{2}\left(\vec{q}_{T}+\vec{q}_{\perp}\right) \Theta\left(q^{-}-Q z_{c}\right) \Theta\left(Q z_{c}-\left(Q-q^{-}\right)\right)\right\}
\end{aligned}
$$

This evaluates to the renormalized result in impact parameter space,

$$
\begin{aligned}
\mathcal{J}_{n}^{\perp(1)}\left(e_{n}, b, \mu, \nu\right)= & -\frac{\alpha_{s} C_{F}}{(2 \pi)^{3}} \ln \frac{\mu^{2}}{E_{J}^{2} e_{n}}\left(2 \ln \frac{1-z_{c}}{z_{c}}-2\left(1-z_{c}\right)+2 z_{c}+\frac{\left(1-z_{c}\right)^{2}}{2}-\frac{z_{c}^{2}}{2}\right) \\
& -\frac{\alpha_{s} C_{F}}{(2 \pi)^{3}} \ln \frac{\mu^{2} b^{2} e^{2 \gamma_{E}}}{4}\left(-2 \ln \frac{\nu}{Q z_{c}}-4 z_{c}-2 \ln \left(1-z_{c}\right)+\frac{z_{c}^{2}}{2}+\frac{1}{2}-\frac{\left(1-z_{c}\right)^{2}}{2}\right)
\end{aligned}
$$


and therefore gives the anomalous dimensions

$$
\begin{gathered}
\gamma_{\mu}^{J}=\frac{\alpha_{s} C_{F}}{\pi}\left(2 \ln \frac{\nu}{Q}+\frac{3}{2}\right) \\
\gamma_{\nu}^{J}=\frac{\alpha_{s} C_{F}}{\pi} \ln \frac{\mu^{2} b^{2} e^{2 \gamma_{E}}}{4}
\end{gathered}
$$

\section{A.3 Soft function}

The soft function that appears in the factorization theorems in eq. (3.29) is defined in eq. (3.30) and it has been calculated in several schemes at higher orders in QCD, as in [97]. and satisfies the following renormalization group equations

$$
\frac{d}{d \ln \mu} S(\mu, \nu)=\gamma^{s}(\mu, \nu) S(\mu, \nu), \quad \frac{d}{d \ln \nu} S(\mu, \nu)=\gamma_{\nu}^{s}(\mu, \nu) \otimes S(\mu, \nu) .
$$

Therefore we find for the one-loop corresponding impact parameter space quantities

$$
S(\mu, \nu)=1+\frac{\alpha_{s}(\mu) C_{i}}{\pi}\left\{4 \ln \left(\frac{\mu_{E}}{\mu}\right) \ln \left(\frac{\nu}{\mu}\right)-2 \ln ^{2}\left(\frac{\mu_{E}}{\mu}\right)-\frac{\pi^{2}}{12}\right\}+\mathcal{O}\left(\alpha_{s}^{2}\right),
$$

with

$$
\gamma^{s}(\mu, \nu)=-4 \frac{\alpha_{s}(\mu) C_{i}}{\pi} \ln \left(\frac{\nu}{\mu}\right)+\mathcal{O}\left(\alpha_{s}^{2}\right), \quad \gamma_{\nu}^{s}(\mu, \nu)=4 \frac{\alpha_{s}(\mu) C_{i}}{\pi} \ln \left(\frac{\mu_{E}}{\mu}\right)+\mathcal{O}\left(\alpha_{s}^{2}\right),
$$

where $\mu_{E}^{-1}=b e^{\gamma_{E}}$ with $b=|\vec{b}|$ is the impact parameter variable conjugate to $\vec{q}_{T}$.

\section{A.4 Resummation}

The vacuum cross section can be resummed by solving the renormalization group equations in $\mu$ and $\nu$ using standard techniques. We will refer the reader to [88] for details on the resummation. In this paper, we are working in a different hierarchy so the details, if not the procedure for resummation, will differ but we postpone presenting the explicit equations till we do a phenomenological study in a future publication.

\section{B Medium jet function}

In this section we present the one loop results for the modified jet function defined in eq. (4.26) as the difference of a real (eq. (4.4)) and virtual (eq. (4.24)) medium jet function.

$$
\begin{aligned}
\mathcal{J}_{n, R}^{A B}\left(e_{n}, \vec{q}_{T}, \vec{k}_{\perp}\right)= & \frac{1}{\mathcal{J}_{n}^{\perp}\left(e_{n}, b\right) k_{\perp}^{2}} \frac{1}{\left[p_{\tilde{X}}^{-}\right]^{2}} \int \frac{d^{2} q_{n} e^{-i \vec{q}_{n} \cdot \vec{b}}}{(2 \pi)^{2}}\left\langle p_{\tilde{X}}-k, \bar{X}_{n}\left|\delta^{2}\left(\mathcal{P}_{\perp}\right) \delta\left(Q-p^{-}\right) \bar{\chi}_{n}(0) \frac{\not h}{2}\right| 0\right\rangle \\
& \times\left\langle 0\left|\chi_{n}(0)\right| p_{\tilde{X}}-k, \bar{X}_{n}\right\rangle\left\langle\tilde{X}_{n}\left|O_{n}^{A}(0)\right| p_{\tilde{X}}-k\right\rangle\left\langle p_{\tilde{X}}-k\left|O_{n}^{B}(0)\right| \tilde{X}_{n}\right\rangle \mathcal{M}_{X_{n}} \\
\mathcal{J}_{n, V}^{A B}(b)= & \frac{1}{k_{\perp}^{2} \mathcal{J}_{n}^{\perp}\left(e_{n}, b\right)} \frac{1}{\left[p_{\tilde{Y}}^{-}\right]^{2}} \int \frac{d^{2} q_{n} e^{-i \vec{q}_{n} \cdot \vec{b}}}{(2 \pi)^{2}}\left\langle p_{\tilde{Y}}+k\left|O_{n}^{A}(0)\right| \tilde{Y}_{n}\right\rangle\left\langle\tilde{Y}_{n}\left|O_{n}^{B}(0)\right| p_{\tilde{Y}}+k\right\rangle \\
& \left\langle p_{\tilde{Y}}+k, \bar{X}_{n}\left|\chi_{n}(0) \frac{\not h}{2}\right| 0\right\rangle\left\langle 0\left|\bar{\chi}_{n}(0) \delta^{2}\left(\mathcal{P}_{\perp}\right) \delta^{2}\left(Q-\mathcal{P}^{-}\right) \mathcal{M}_{V}\right| p_{\tilde{Y}}+k, \bar{X}_{n}\right\rangle
\end{aligned}
$$


and the medium jet function

$$
\mathcal{J}_{n, \text { Med }}^{A B}=\mathcal{J}_{n, R}^{A B}-\mathcal{J}_{n, V}^{A B}
$$

$\mathcal{J}_{n}^{\perp}\left(e_{n}, b\right)$ being the vacuum jet function.

The complete one loop corrections include corrections to the Glauber vertex and the corrections to the hard vertex(SCET current). In this paper, we will only consider one loop corrections for the hard vertex which correspond to elastic collisions with the medium. Both of these corrections are equally important and will be needed for any phenomenological analysis.

Therefore, we will evaluate the Glauber collinear current to tree level. The Glauber collinear currents $\left(O_{n}^{A}, O_{n}^{B}\right)$ can be either quark or gluon currents, so we need to consider both cases. However, there will be no contributions proportional to t from the interference term between these currents so we can write the one loop corrections as a sum over the quark and gluon terms.

Quark operator. We begin with the quark operator $O_{n}^{A}=\bar{\chi}_{n} W_{n}^{\dagger} \frac{\hbar}{2} T^{A} W_{n} \chi_{n}$.

For the quark operator, this will create and annihilate a single quark at tree level so that $\left|\tilde{X}_{n}\right\rangle \equiv\left|X_{1}\right\rangle$ and $\bar{X}_{n} \equiv\left|X_{i}\right\rangle$, with $i \in\{2, \infty\}$.

We can separately consider the contribution from the Real and virtual medium jet functions and combine them later.

$$
\begin{aligned}
\mathcal{J}_{n, R}^{A B}\left(e_{n}, k\right)= & \frac{1}{\mathcal{J}_{n}^{\perp}\left(e_{n}, b\right) k_{\perp}^{2}} \int \frac{d^{2} q_{n} e^{-i \vec{q}_{n} \cdot \vec{b}}}{(2 \pi)^{2}} \Theta\left(e_{n}-\frac{8}{Q^{2}} \sum_{i, j \in \mathrm{gr}} p_{X i} \cdot p_{X j}\right) \delta^{2}\left(\vec{q}_{n}-\sum_{i \notin \mathrm{gr}} \vec{p}_{X i}^{\perp}-\vec{k}_{\perp}\right) \\
& \operatorname{Tr}\left[T^{A} T^{B}\left\langle p_{X_{1}}-k, p_{X i}\left|\delta^{2}\left(\mathcal{P}_{\perp}\right) \delta\left(Q-p^{-}\right) \bar{\chi}_{n}(0) \frac{\not h}{2}\right| 0\right\rangle\left\langle 0\left|\chi_{n}(0)\right| p_{X_{1}}-k, p_{X i}\right\rangle\right]
\end{aligned}
$$

we can make a change of variables taking $p_{X_{1}}-k \rightarrow p_{X_{1}}$. We can rearrange the measurement function for the jet mass as

$$
M=\sum_{i, j} p_{X i} \cdot p_{X j}=\sum_{j \neq 1} p_{X 1} \cdot p_{X j}+\sum_{i, j \neq 1} p_{X i} \cdot p_{X j} \rightarrow \sum_{i, j} p_{X i} \cdot p_{X j}+\sum_{j} k \cdot p_{X j}-k \cdot p_{X_{1}}
$$

Now using the fact that $\sum_{j} p_{X j}^{-}=Q, \sum_{j} \vec{p}_{X j}^{\perp}=0$ and using the power counting of our Glauber mode,

$$
M=\sum_{i, j} p_{X i} \cdot p_{X j}+\frac{1}{2} k^{+} Q-\frac{1}{2} k^{+} p_{X_{1}}^{-}+\vec{k}_{\perp} \cdot p_{X_{1}}^{\perp}
$$

Given the on-shell condition on $p_{X_{1}}-k$ from eq. (B.4),

$$
\begin{aligned}
k^{+} p_{X}^{-} & =2 \vec{p}_{X_{1}}^{\perp} \cdot \vec{k}_{\perp}+\vec{k}_{\perp}^{2} \\
M & =\sum_{i, j} p_{X i} \cdot p_{X j}+\frac{Q}{2 p_{X_{1}}^{-}} 2 \vec{p}_{X_{1}}^{\perp} \cdot \vec{k}_{\perp}+\frac{\vec{k}_{\perp}^{2}}{2 p_{X_{1}}^{-}}\left(Q-p_{X_{1}}^{-}\right)
\end{aligned}
$$


so that our jet function becomes

$$
\begin{aligned}
\mathcal{J}^{A B}\left(e_{n}, k\right) \\
=\frac{1}{\mathcal{J}_{n}^{\perp}\left(e_{n}, b\right) k_{\perp}^{2}} \int \frac{d^{2} q_{n} e^{-i \vec{q}_{n} \cdot \vec{b}}}{(2 \pi)^{2}} \Theta\left(e_{n}-\left[8 \frac{\vec{k}_{\perp} \cdot p_{X_{1}}^{\perp}}{Q p_{X_{1}}^{-}}+8 \frac{k_{\perp}^{2}}{2 p_{X}^{-} Q^{2}}\left(Q-p_{X_{1}}^{-}\right)\right]_{X_{1} \in \mathrm{gr}}\right. \\
\left.-\frac{8}{Q^{2}} \sum_{i, j \in \mathrm{gr}} p_{X i} \cdot p_{X j}\right) \delta^{2}\left(\vec{q}_{n}-\sum_{i \notin \mathrm{gr}} \vec{p}_{X i, \perp}-\left.\vec{k}_{\perp}\right|_{X_{1} \in \mathrm{gr}}\right)\left\langle X_{i}\left|\delta^{2}\left(\mathcal{P}_{\perp}\right) \delta\left(Q-p^{-}\right) \bar{\chi}_{n}(0) \frac{\hbar_{n}}{2}\right| 0\right\rangle\left\langle 0\left|\chi_{n}(0)\right| X_{i}\right\rangle
\end{aligned}
$$

So the definition of the jet mass measurement is altered from its standard definition by a function of $k_{\perp}$ due to the interaction of the parton $X_{1}$ with the medium. Even though we have worked this out for the case of jet mass measurement, this is a general property for all jet substructure observables. $p_{X_{1}}$ here is the momentum of a quark so that when we compute the jet function, it will automatically sum over all the quarks in the jet as it should. When we include the gluon-medium interaction, we will have a similar sum over gluon states.

$$
\mathcal{J}_{n, R}^{A B,(0)}\left(e_{n}, \vec{k}_{\perp}, \vec{b}\right)=\frac{\frac{1}{2} \delta^{A B}}{k_{\perp}^{2}} e^{-i \vec{k}_{\perp} \cdot \vec{b}}
$$

The vacuum jet function which appears in the denominator is just 1 at tree level.

At one loop, I have both real and virtual diagrams. The gluon emitted as a radiative correction interacts with the medium and acquires a mass $m_{D}$ with the hierarchy $m_{D} \ll e_{n}$ since we are working in a weak coupling regime. If the jet function is IR finite, then the $m_{D}$ scale is irrelevant and we can use dimensional regularization which does not distinguish between UV and IR divergences. However, we expect that the medium will induce nontrivial Infra-Red physics of this function, it is no longer guaranteed that this function is IR finite, in the sense that it can be sensitive to scale $m_{D}$. We are dividing out by the vacuum jet function which, at one loop is equivalent to subtracting the one loop result in impact parameter(b) space. This immediately tells us that the virtual diagrams simply cancel out between the numerator and denominator and the answer will just be the difference between the real diagrams which we now consider. There are two Feynman diagram that contribute as shown in figure 3 .

$$
\mathcal{J}_{n, R}^{A B,(1)}=\frac{\frac{1}{2} \delta^{A B}}{k_{\perp}^{2}}\left\{\left(R_{a}(\vec{b})-e^{-i \vec{k}_{\perp} \cdot \vec{b}} R_{a}^{\operatorname{Vac}}(\vec{b})\right)+\left(R_{b}(\vec{b})-e^{-i \vec{k}_{\perp} \cdot \vec{b}} R_{b}^{\mathrm{Vac}}(\vec{b})\right)\right\} \equiv \frac{\frac{1}{2} \delta^{A B}}{k_{\perp}^{2}} R(b)
$$

Depending on which partons pass the grooming condition, we have three contributions just as for the vacuum diagram. For the diagram (a), we have

$$
\begin{aligned}
R_{a}= & 8 g^{2} C_{F} \int \frac{d^{2} q_{n}}{(2 \pi)^{2}} e^{-i \vec{q}_{n} \cdot \vec{b}} \int d^{4} p \delta^{+}\left(p^{2}\right) \int \frac{d^{4} q}{(2 \pi)^{3}} \delta^{+}\left(q^{2}-m_{D}^{2}\right) \delta\left(Q-p^{-}-q^{-}\right) \delta^{2}\left(\vec{q}_{\perp}+\vec{p}_{\perp}\right) \frac{p^{-}\left(p^{-}+q^{-}\right)}{q^{-}(p+q)^{2}} \\
& \left\{\Theta\left(e_{n}-8 \frac{\vec{k}_{\perp} \cdot \vec{p}_{\perp}}{Q p^{-}}-8 \frac{k_{\perp}^{2}\left(Q-p^{-}\right)}{2 p^{-} Q^{2}}-\frac{8}{2 Q}\left(p^{+}+q^{+}\right)\right) \delta^{2}\left(\vec{q}_{n}-\vec{k}_{\perp}\right) \Theta\left(q^{-}-Q z_{c}\right) \Theta\left(p^{-}-Q z_{c}\right)\right. \\
& \left.+\Theta\left(Q z_{c}-q^{-}\right) \Theta\left(p^{-}-Q z_{c}\right) \delta^{2}\left(\vec{q}_{n}-\vec{q}_{\perp}-\vec{k}_{\perp}\right)+\Theta\left(Q z_{c}-p^{-}\right) \Theta\left(q^{-}-Q z_{c}\right) \delta^{2}\left(\vec{q}_{n}-\vec{p}_{\perp}\right)\right\}
\end{aligned}
$$




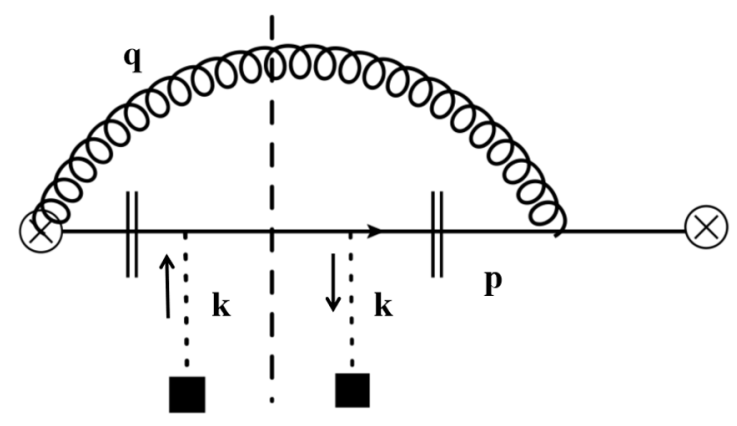

(a)

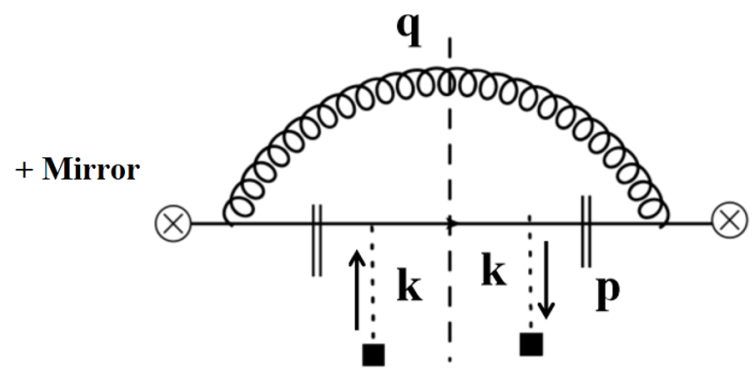

(b)

Figure 3. Real emission diagrams for the medium induced jet function. The $\otimes$ is the hard vertex that creates the quark. The Gluon in diagram (a) is from the $\mathrm{n}$ collinear Wilson line attached to the hard vertex. The dotted line indicates the Glauber gluon exchange with the medium. We also indicate that the quark goes on-shell before interacting with the medium. Diagram (b) comes from insertions of $H_{n, \text { int }}$ which describes interactions between n collinear partons.

we can shift $\vec{q}_{n} \rightarrow \vec{q}_{n}+\vec{k}_{\perp}$ and subtract the corresponding vacuum diagram in figure 2 . Doing the same for diagram (b), we get

$$
\begin{aligned}
R= & 4 g^{2} C_{F} \int \frac{d^{2} q_{n}}{(2 \pi)^{2}} e^{-i\left(\vec{q}_{n}+\vec{k}_{\perp}\right) \cdot \vec{b}} \int \frac{d^{4} q}{(2 \pi)^{3}} \delta^{+}\left(q^{2}-m_{D}^{2}\right)\left\{\frac{\left(Q-q^{-}\right)}{q^{-}\left(q^{+}+\frac{q_{\perp}^{2}}{Q-q^{-}}\right)}+\frac{q_{\perp}^{2}}{2\left(Q-q^{-}\right)^{2}\left(q^{+}+\frac{q_{\perp}^{2}}{Q-q^{-}}\right)^{2}}\right\} \\
& \left\{\left(\Theta\left(e_{n}-8 \frac{\vec{k}_{\perp} \cdot \vec{p}_{\perp}}{Q p^{-}}-8 \frac{k_{\perp}^{2}\left(Q-p^{-}\right)}{2 p^{-} Q^{2}}-\frac{8}{2 Q}\left(\frac{q_{\perp}^{2}}{Q-q^{-}}+q^{+}\right)\right)-\Theta\left(e_{n}-\frac{8}{2 Q}\left(\frac{q_{\perp}^{2}}{Q-q^{-}}+q^{+}\right)\right)\right)\right. \\
& \left.\delta^{2}\left(\vec{q}_{n}\right) \Theta\left(q^{-}-Q z_{c}\right) \Theta\left(p^{-}-Q z_{c}\right)+\Theta\left(Q z_{c}-p^{-}\right) \Theta\left(q^{-}-Q z_{c}\right)\left[\delta^{2}\left(\vec{q}_{n}+\vec{k}_{\perp}+\vec{q}_{\perp}\right)-\delta^{2}\left(\vec{q}_{n}+\vec{q}_{\perp}\right)\right]\right\}
\end{aligned}
$$

The other piece of the medium jet function is $\mathcal{J}_{n, V}^{A B}$ defined in eq. (B.2). Once again we consider the quark operator and evaluate the Glauber current at tree level, which leads to

$$
\begin{aligned}
\mathcal{J}_{n, V}^{A B}(b)= & \frac{\frac{1}{2} \delta^{A B}}{k_{\perp}^{2} \mathcal{J}_{n}^{\perp}\left(e_{n}, b\right)} \int \frac{d^{2} q_{n} e^{-i \vec{q}_{n} \cdot \vec{b}}}{(2 \pi)^{2}}\left\langle p_{\tilde{Y}}+k, \bar{X}_{n}\left|\chi_{n}(0) \frac{\not h}{2}\right| 0\right\rangle \\
& \left\langle 0\left|\bar{\chi}_{n}(0) \delta^{2}\left(\mathcal{P}^{\perp}\right) \delta^{2}\left(Q-\mathcal{P}^{-}\right) \mathcal{M}_{V}\right| p_{\tilde{Y}}+k, \bar{X}_{n}\right\rangle \\
= & \frac{\frac{1}{2} \delta^{A B}}{k_{\perp}^{2}}
\end{aligned}
$$

where the numerator is simply proportional to the vacuum jet function and hence cancels out with the denominator leaving behind a very simple result. Therefore, for the Quark Glauber operator, our result is

$$
\mathcal{J}_{n, \text { Med }}^{A B, q}=\frac{\frac{1}{2} \delta^{A B}}{k_{\perp}^{2}}\left\{e^{-i \vec{k}_{\perp} \cdot \vec{b}}-1+R(b)\right\}
$$


$\mathrm{R}(\mathrm{b})$ evaluates to

$$
\begin{aligned}
R= & \frac{\alpha_{s} C_{F} e^{-i \vec{k}_{\perp} \cdot \vec{b}}}{2 \pi}\left[\int_{z_{c}}^{1-z_{c}} d z p_{g q}(z)\left\{\Theta\left(z_{c}-\frac{y}{e_{n}+y}\right) \ln \left(\frac{-B(z)}{e_{n}(1-z)}\right)-\Theta\left(z_{c}-\frac{e_{n}}{e_{n}+y}\right) \ln \frac{B(z) e_{n}}{y M}\right\}\right. \\
& \left.+\Theta\left(\frac{e_{n}}{e_{n}+y}-z_{c}\right) \Theta\left(\frac{y}{e_{n}+y}-z_{c}\right)\left\{\int_{z_{c}}^{\frac{e_{n}}{e_{n}+y}} d z p_{g q}(z) \ln \left(\frac{-B(z)}{e_{n}(1-z)}\right)-\int_{\frac{e_{n}}{e_{n}+y}}^{1-z_{c}} d z p_{g q}(z) \ln \frac{B(z) e_{n}}{y M}\right\}\right] \\
& +\frac{\alpha_{s} C_{F}}{2 \pi}\left(e^{-i \vec{k}_{\perp} \cdot \vec{b}}-1\right) \int_{1-z_{c}}^{1} p_{g q}(z) \ln \frac{m_{D}^{2} b^{2} e^{2 \gamma_{E}}(1-z)}{4}
\end{aligned}
$$

where

$$
p_{g q}(z)=\frac{1+(1-z)^{2}}{z}, \quad B(z)=z y-e_{n}(1-z), \quad y=\frac{4 k_{\perp}^{2}}{Q^{2}}, \quad M=\frac{4 m_{D}^{2}}{Q^{2}}
$$

The integral over z can be done analytically with little difficulty. However, the resulting final expression is long and not very illuminating, so we present the result as an integral over z. The result is finite and there are no UV or rapidity divergences. However, it is sensitive to the IR cut-off $m_{D}$.

Gluon operator. We will now consider the contribution to the one loop modified jet function from the gluon operator $O_{n, g}^{A}=\frac{i}{2} f^{A B C} \mathcal{B}_{n \perp, \mu}^{B} \frac{\bar{n}}{2} \cdot\left(\mathcal{P}+\mathcal{P}^{\dagger}\right) \mathcal{B}_{n \perp}^{C \mu}$, which creates and annihilates a single gluon at tree level.

Following the same series of steps as for the quark operator, we arrive at

$$
\begin{aligned}
\mathcal{J}_{n, R}^{A B, g}\left(e_{n}, \vec{k}_{\perp}\right) \\
=\frac{1 / 2}{k_{\perp}^{2} \mathcal{J}_{n}^{\perp}\left(e_{n}, b\right)} \int \frac{d^{2} q_{n} e^{-i \vec{q}_{n} \cdot \vec{b}}}{(2 \pi)^{2}} \Theta\left(e_{n}-\left[8 \frac{\vec{k}_{\perp} \cdot p_{X_{1}}^{\perp}}{Q_{X_{1}}^{-}}+8 \frac{k_{\perp}^{2}}{2 p_{X_{1}}^{-} Q^{2}}\left(Q-p_{X_{1}}^{-}\right)\right]_{X_{1} \in \mathrm{gr}}\right. \\
\left.\quad-\frac{8}{Q^{2}} \sum_{i, j \in \mathrm{gr}} p_{X i} \cdot p_{X j}\right) \delta^{2}\left(\vec{q}_{n}-\sum_{i \notin \mathrm{gr}} \vec{p}_{X i}^{\perp}-\left.\vec{k}_{\perp}\right|_{X_{1} \in \mathrm{gr}}\right)\left\langle p_{X i}\left|\delta^{2}\left(\mathcal{P}^{\perp}\right) \delta\left(Q-p^{-}\right) \bar{\chi}_{n}(0) \frac{\hbar}{2}\right| 0\right\rangle\left\langle 0\left|\chi_{n}(0)\right| p_{X i}\right\rangle \\
\quad \times \frac{f^{A b c} f^{B b c} \epsilon^{\mu}\left(p_{X_{1}}\right) \epsilon^{* \nu}\left(p_{X_{1}}\right)}{\left(p_{X_{1}}^{-}\right)^{2}}\left[\left(p_{X_{1}}^{-}\right)^{2} g_{\perp}^{\mu \nu}-\bar{n}^{\mu} p_{X_{1}}^{\perp \nu} p_{X_{1}}^{-}-\bar{n}^{\mu} \bar{n}^{\nu} p_{X_{1}, \perp}^{2}-\bar{n}^{\nu} p_{X_{1}}^{-} p_{X_{1} \perp}^{\mu}\right]
\end{aligned}
$$

where $p_{X_{1}}$ is now the momentum of a gluon. The contribution from the gluon operator only starts at $\mathrm{O}\left(\alpha_{s}\right)$, hence for a one loop calculation, we can evaluate the vacuum $\mathcal{J}_{n}^{\perp}\left(e_{n}, b\right)$ in the denominator to tree level.

$$
\mathcal{J}_{n, R}^{A B, g(1)}\left(e_{n}, \vec{k}_{\perp}, b\right)=\frac{\frac{1}{2} \delta^{A B}}{k_{\perp}^{2}} R^{g}
$$

We can now compute the one loop results which correspond to the diagram figure 4. However, 

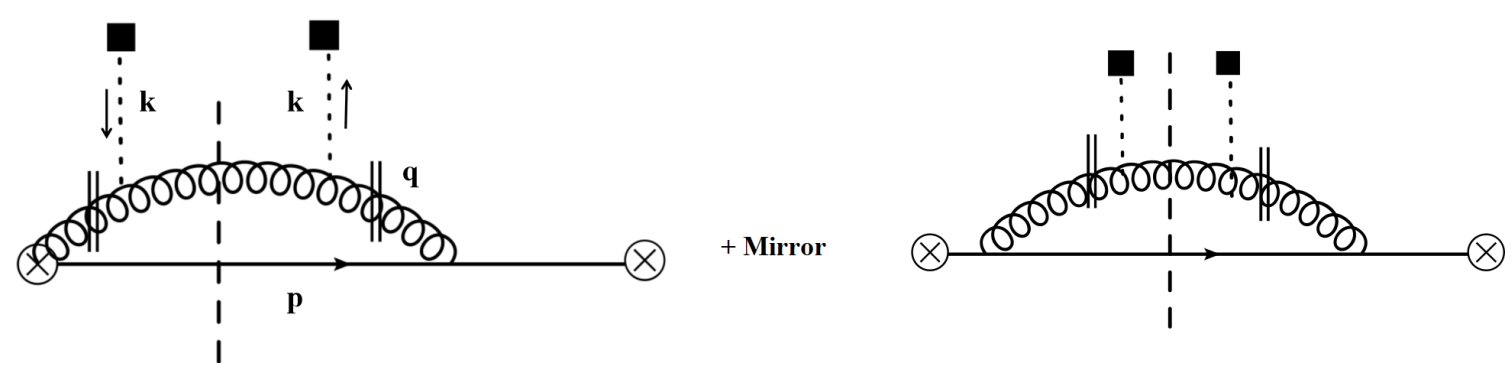

Figure 4. Gluon real interaction with the medium.

diagram (a) reduces to 0 and we only get a contribution form (b). This gives us

$$
\begin{aligned}
R_{g}= & 4 g^{2} N_{c} \int \frac{d^{2} q_{n}}{(2 \pi)^{2}} e^{-i \vec{q}_{n} \cdot \vec{b}} \int d^{4} p \delta^{+}\left(p^{2}\right) \int \frac{d^{4} q}{(2 \pi)^{3}} \\
& \delta^{+}\left(q^{2}-m_{D}^{2}\right) \delta\left(Q-p^{-}-q^{-}\right) \delta^{2}\left(\vec{q}_{\perp}+\vec{p}_{\perp}\right) \frac{Q^{2}+\left(p^{-}\right)^{2}}{p^{-}\left(q^{-}\right)^{2}\left[(p+q)^{2}\right]^{2}} \\
& \left\{\Theta\left(e_{n}-8 \frac{\vec{k}_{\perp} \cdot \vec{q}_{\perp}}{Q q^{-}}-8 \frac{k_{\perp}^{2}\left(Q-q^{-}\right)}{2 q^{-} Q^{2}}-\frac{8}{2 Q}\left(p^{+}+q^{+}\right)\right) \delta^{2}\left(\vec{q}_{n}-\vec{k}_{\perp}\right) \Theta\left(q^{-}-Q z_{c}\right) \Theta\left(p^{-}-Q z_{c}\right)\right. \\
& \left.+\Theta\left(Q z_{c}-q^{-}\right) \Theta\left(p^{-}-Q z_{c}\right) \delta^{2}\left(\vec{q}_{n}-\vec{q}_{\perp}\right)+\Theta\left(Q z_{c}-p^{-}\right) \Theta\left(q^{-}-Q z_{c}\right) \delta^{2}\left(\vec{q}_{n}-\vec{p}_{\perp}-\vec{k}_{\perp}\right)\right\}
\end{aligned}
$$

We can now do a similar analysis for the gluon operator contribution to $\mathcal{J}_{n, V}^{A B}$ which at one loop yields

$$
\mathcal{J}_{n, V}^{A B, g(1)}\left(e_{n}, \vec{k}_{\perp}, b\right)=\frac{\frac{1}{2} \delta^{A B}}{k_{\perp}^{2}} V^{g}
$$

These are the diagrams where the Glauber insertion happens on the same side of the cut as shown in figure 5. Given that the gluon is on-shell, this will merely give us the real vacuum diagram dressed with the virtual interaction of the gluon with the medium.

$$
\begin{aligned}
V_{g}= & 4 g^{2} N_{c} \int \frac{d^{2} q_{n}}{(2 \pi)^{2}} e^{-i \vec{q}_{n} \cdot \vec{b}} \int d^{4} p \delta^{+}\left(p^{2}\right) \int \frac{d^{4} q}{(2 \pi)^{3}} \delta^{+}\left(q^{2}-m_{g}^{2}\right) \delta\left(Q-p^{-}-q^{-}\right) \delta^{2}\left(\vec{q}_{\perp}+\vec{p}_{\perp}\right) \\
& \frac{Q^{2}+\left(p^{-}\right)^{2}}{p^{-}\left(q^{-}\right)^{2}\left[(p+q)^{2}\right]^{2}}\left\{\Theta\left(e_{n}-\frac{8}{2 Q}\left(p^{+}+q^{+}\right)\right) \delta^{2}\left(\vec{q}_{n}\right) \Theta\left(q^{-}-Q z_{c}\right) \Theta\left(p^{-}-Q z_{c}\right)\right. \\
& \left.+\Theta\left(Q z_{c}-q^{-}\right) \Theta\left(p^{-}-Q z_{c}\right) \delta^{2}\left(\vec{q}_{n}-\vec{q}_{\perp}\right)+\Theta\left(Q z_{c}-p^{-}\right) \Theta\left(q^{-}-Q z_{c}\right) \delta^{2}\left(\vec{q}_{n}-\vec{p}_{\perp}\right)\right\}
\end{aligned}
$$

which is just the same as $R_{g}$ (eq. (B.17)) in the limit $\vec{k}_{\perp} \rightarrow 0$. We can then combine the two terms to give us the full contribution from the gluon current

$$
\mathcal{J}_{n, \text { Med }}^{A B, g}\left(e_{n}, \vec{k}_{\perp}, b\right)=\frac{\frac{1}{2} \delta^{A B}}{k_{\perp}^{2}}\left\{R_{g}-V_{g}\right\}
$$



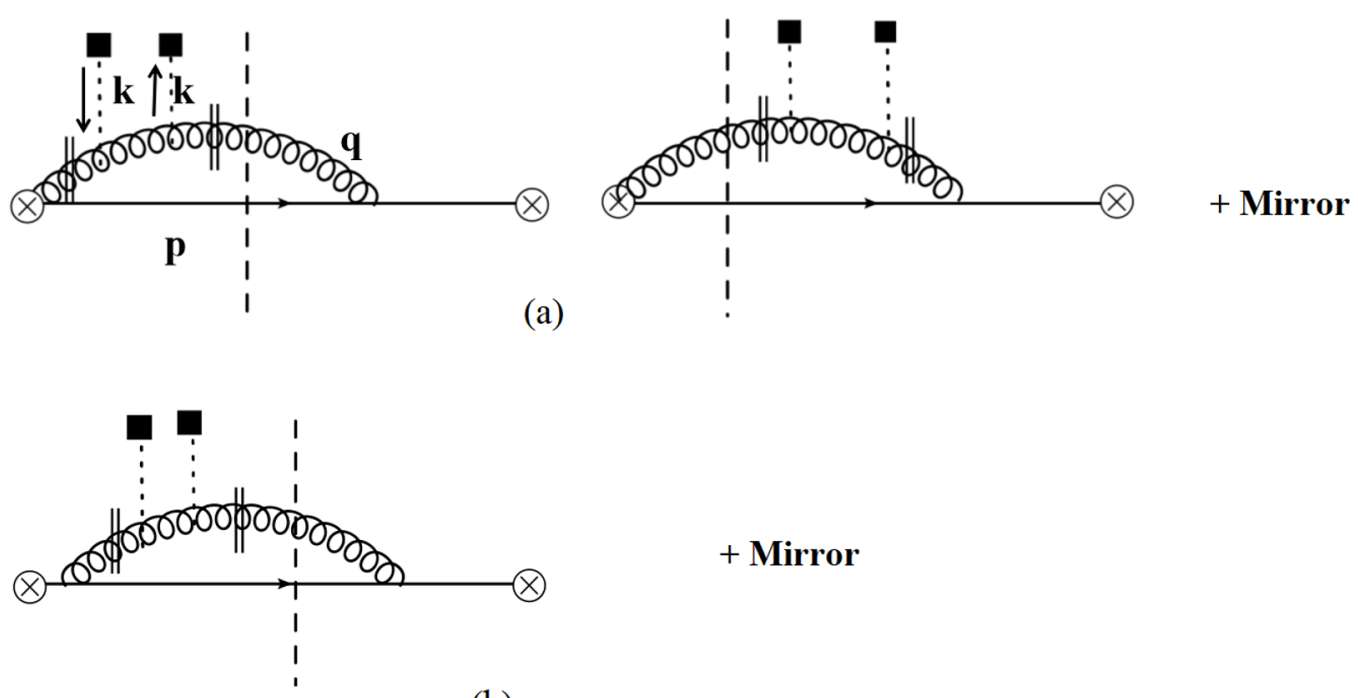

\section{+ Mirror}

(b)

Figure 5. Gluon virtual interaction with the medium.

where $R_{g}$ evaluates to

$$
\begin{aligned}
R_{g}= & \frac{\alpha_{s} N_{c} e^{-i \vec{k}_{\perp} \cdot \vec{b}}}{2 \pi}\left[\int_{z_{c}}^{1-z_{c}} d z p_{g q}(z)\left\{\Theta\left(z_{c}-\frac{y}{e_{n}+y}\right) \ln \frac{A(z)}{M}-\Theta\left(z_{c}-\frac{e_{n}}{e_{n}+y}\right) \ln \left(\frac{-A(z)}{y(1-z)}\right)\right\}\right. \\
& \left.-\Theta\left(\frac{e_{n}}{e_{n}+y}-z_{c}\right) \Theta\left(\frac{y}{e_{n}+y}-z_{c}\right)\left\{\int_{z_{c}}^{\frac{y}{e_{n}+y}} d z p_{g q}(z) \ln \left(\frac{-A(z)}{y(1-z)}\right)-\int_{\frac{y}{e_{n}+y}}^{1-z_{c}} d z p_{g q}(z) \ln \frac{A(z)}{M}\right\}\right] \\
& -\frac{\alpha_{s} N_{c}}{2 \pi} e^{-i \vec{k}_{\perp} \cdot \vec{b}} \int_{1-z_{c}}^{1} p_{g q}(z) \ln \frac{m_{D}^{2} b^{2} e^{2 \gamma_{E}}(1-z)}{4}
\end{aligned}
$$

which again is sensitive to $\ln m_{D}$ and

$$
A(z)=e_{n} z-(1-z) y
$$

$V_{g}$ is obtained by simply evaluating $R_{g}$ at $k_{\perp}=0$.

Open Access. This article is distributed under the terms of the Creative Commons Attribution License (CC-BY 4.0), which permits any use, distribution and reproduction in any medium, provided the original author(s) and source are credited.

\section{References}

[1] M. Gyulassy and X.-n. Wang, Multiple collisions and induced gluon Bremsstrahlung in QCD, Nucl. Phys. B 420 (1994) 583 [nucl-th/9306003] [inSPIRE].

[2] X.-N. Wang, M. Gyulassy and M. Plumer, The LPM effect in QCD and radiative energy loss in a quark gluon plasma, Phys. Rev. D 51 (1995) 3436 [hep-ph/9408344] [INSPIRE].

[3] R. Baier, Y.L. Dokshitzer, S. Peigne and D. Schiff, Induced gluon radiation in a QCD medium, Phys. Lett. B 345 (1995) 277 [hep-ph/9411409] [INSPIRE]. 
[4] R. Baier, Y.L. Dokshitzer, A.H. Mueller, S. Peigne and D. Schiff, Radiative energy loss of high-energy quarks and gluons in a finite volume quark-gluon plasma, Nucl. Phys. B $\mathbf{4 8 3}$ (1997) 291 [hep-ph/9607355] [INSPIRE].

[5] R. Baier, Y.L. Dokshitzer, A.H. Mueller, S. Peigne and D. Schiff, Radiative energy loss and $p_{T}$ broadening of high-energy partons in nuclei, Nucl. Phys. B 484 (1997) 265 [hep-ph/9608322] [INSPIRE].

[6] B.G. Zakharov, Fully quantum treatment of the Landau-Pomeranchuk-Migdal effect in QED and QCD, JETP Lett. 63 (1996) 952 [hep-ph/9607440] [INSPIRE].

[7] B.G. Zakharov, Radiative energy loss of high-energy quarks in finite size nuclear matter and quark-gluon plasma, JETP Lett. 65 (1997) 615 [hep-ph/9704255] [INSPIRE].

[8] M. Gyulassy, P. Levai and I. Vitev, Jet quenching in thin quark gluon plasmas. 1. Formalism, Nucl. Phys. B 571 (2000) 197 [hep-ph/9907461] [INSPIRE].

[9] M. Gyulassy, P. Levai and I. Vitev, Reaction operator approach to nonAbelian energy loss, Nucl. Phys. B 594 (2001) 371 [nucl-th/0006010] [INSPIRE].

[10] U.A. Wiedemann, Gluon radiation off hard quarks in a nuclear environment: Opacity expansion, Nucl. Phys. B 588 (2000) 303 [hep-ph/0005129] [INSPIRE].

[11] X.-f. Guo and X.-N. Wang, Multiple scattering, parton energy loss and modified fragmentation functions in deeply inelastic e A scattering, Phys. Rev. Lett. 85 (2000) 3591 [hep-ph/0005044] [INSPIRE].

[12] X.-N. Wang and X.-f. Guo, Multiple parton scattering in nuclei: Parton energy loss, Nucl. Phys. A 696 (2001) 788 [hep-ph/0102230] [INSPIRE].

[13] P.B. Arnold, G.D. Moore and L.G. Yaffe, Photon and gluon emission in relativistic plasmas, JHEP 06 (2002) 030 [hep-ph/0204343] [INSPIRE].

[14] P.B. Arnold, G.D. Moore and L.G. Yaffe, Effective kinetic theory for high temperature gauge theories, JHEP 01 (2003) 030 [hep-ph/0209353] [INSPIRE].

[15] C.A. Salgado and U.A. Wiedemann, Calculating quenching weights, Phys. Rev. D 68 (2003) 014008 [hep-ph/0302184] [INSPIRE].

[16] N. Armesto, C.A. Salgado and U.A. Wiedemann, Medium induced gluon radiation off massive quarks fills the dead cone, Phys. Rev. D 69 (2004) 114003 [hep-ph/0312106] [INSPIRE].

[17] A. Majumder, B. Müller and S.A. Bass, Longitudinal Broadening of Quenched Jets in Turbulent Color Fields, Phys. Rev. Lett. 99 (2007) 042301 [hep-ph/0611135] [INSPIRE].

[18] A. Majumder, B. Müller and X.-N. Wang, Small shear viscosity of a quark-gluon plasma implies strong jet quenching, Phys. Rev. Lett. 99 (2007) 192301 [hep-ph/0703082] [INSPIRE].

[19] R.B. Neufeld, B. Müller and J. Ruppert, Sonic Mach Cones Induced by Fast Partons in a Perturbative Quark-Gluon Plasma, Phys. Rev. C 78 (2008) 041901 [arXiv:0802.2254] [INSPIRE].

[20] R.B. Neufeld and B. Müller, The sound produced by a fast parton in the quark-gluon plasma is a 'crescendo', Phys. Rev. Lett. 103 (2009) 042301 [arXiv:0902.2950] [InSPIRE].

[21] BRAHMS collaboration, Quark gluon plasma and color glass condensate at RHIC? The Perspective from the BRAHMS experiment, Nucl. Phys. A 757 (2005) 1 [nucl-ex/0410020] [INSPIRE]. 
[22] PHOBOS collaboration, The PHOBOS perspective on discoveries at RHIC, Nucl. Phys. A 757 (2005) 28 [nucl-ex/0410022] [INSPIRE].

[23] STAR collaboration, Experimental and theoretical challenges in the search for the quark gluon plasma: The STAR Collaboration's critical assessment of the evidence from RHIC collisions, Nucl. Phys. A 757 (2005) 102 [nucl-ex/0501009] [InSPIRE].

[24] PHENIX collaboration, Formation of dense partonic matter in relativistic nucleus-nucleus collisions at RHIC: Experimental evaluation by the PHENIX collaboration, Nucl. Phys. A $\mathbf{7 5 7}$ (2005) 184 [nucl-ex/0410003] [INSPIRE].

[25] ATLAS collaboration, Observation of a Centrality-Dependent Dijet Asymmetry in Lead-Lead Collisions at $\sqrt{s_{N N}}=2.77 \mathrm{TeV}$ with the ATLAS Detector at the LHC, Phys. Rev. Lett. 105 (2010) 252303 [arXiv:1011.6182] [INSPIRE].

[26] ALICE collaboration, Suppression of Charged Particle Production at Large Transverse Momentum in Central Pb-Pb Collisions at $\sqrt{s_{N N}}=2.76$ TeV, Phys. Lett. B 696 (2011) 30 [arXiv: 1012.1004] [INSPIRE].

[27] CMS collaboration, Observation and studies of jet quenching in PbPb collisions at nucleon-nucleon center-of-mass energy $=2.76$ TeV, Phys. Rev. C 84 (2011) 024906 [arXiv: 1102.1957] [INSPIRE].

[28] ATLAS collaboration, Measurements of the Nuclear Modification Factor for Jets in $\mathrm{Pb}+\mathrm{Pb}$ Collisions at $\sqrt{s_{\mathrm{NN}}}=2.76 \mathrm{TeV}$ with the ATLAS Detector, Phys. Rev. Lett. 114 (2015) 072302 [arXiv: 1411.2357] [INSPIRE].

[29] ALICE collaboration, Measurement of jet suppression in central $\mathrm{Pb}-\mathrm{Pb}$ collisions at $\sqrt{s_{\mathrm{NN}}}=2.76 \mathrm{TeV}$, Phys. Lett. B 746 (2015) 1 [arXiv:1502.01689] [INSPIRE].

[30] CMS collaboration, Measurement of inclusive jet cross sections in pp and $\mathrm{PbPb}$ collisions at $\sqrt{s_{N N}}=2.76 \mathrm{TeV}$, Phys. Rev. C 96 (2017) 015202 [arXiv: 1609.05383] [INSPIRE].

[31] Y. Mehtar-Tani, J.G. Milhano and K. Tywoniuk, Jet physics in heavy-ion collisions, Int. J. Mod. Phys. A 28 (2013) 1340013 [arXiv:1302.2579] [INSPIRE].

[32] J.-P. Blaizot and Y. Mehtar-Tani, Jet Structure in Heavy Ion Collisions, Int. J. Mod. Phys. E 24 (2015) 1530012 [arXiv: 1503.05958] [INSPIRE].

[33] G.-Y. Qin and X.-N. Wang, Jet quenching in high-energy heavy-ion collisions, Int. J. Mod. Phys. E 24 (2015) 1530014 [arXiv:1511.00790] [InSPIRE].

[34] S. Cao and X.-N. Wang, Jet quenching and medium response in high-energy heavy-ion collisions: a review, Rept. Prog. Phys. 84 (2021) 024301 [arXiv:2002.04028] [INSPIRE].

[35] J. Casalderrey-Solana, D.C. Gulhan, J.G. Milhano, D. Pablos and K. Rajagopal, A Hybrid Strong/Weak Coupling Approach to Jet Quenching, JHEP 10 (2014) 019 [Erratum ibid. 09 (2015) 175] [arXiv: 1405.3864] [INSPIRE].

[36] J. Casalderrey-Solana, D.C. Gulhan, J.G. Milhano, D. Pablos and K. Rajagopal, Predictions for Boson-Jet Observables and Fragmentation Function Ratios from a Hybrid Strong/Weak Coupling Model for Jet Quenching, JHEP 03 (2016) 053 [arXiv: 1508.00815] [INSPIRE].

[37] Z. Hulcher, D. Pablos and K. Rajagopal, Resolution Effects in the Hybrid Strong/Weak Coupling Model, JHEP 03 (2018) 010 [arXiv:1707.05245] [INSPIRE].

[38] J. Casalderrey-Solana, Z. Hulcher, G. Milhano, D. Pablos and K. Rajagopal, Simultaneous description of hadron and jet suppression in heavy-ion collisions, Phys. Rev. C 99 (2019) 051901 [arXiv: 1808.07386] [INSPIRE]. 
[39] J. Casalderrey-Solana, G. Milhano, D. Pablos and K. Rajagopal, Modification of Jet Substructure in Heavy Ion Collisions as a Probe of the Resolution Length of Quark-Gluon Plasma, JHEP 01 (2020) 044 [arXiv: 1907.11248] [INSPIRE].

[40] H. Liu, K. Rajagopal and U.A. Wiedemann, Calculating the jet quenching parameter from AdS/CFT, Phys. Rev. Lett. 97 (2006) 182301 [hep-ph/0605178] [INSPIRE].

[41] P.C. Argyres, M. Edalati and J.F. Vazquez-Poritz, Spacelike strings and jet quenching from a Wilson loop, JHEP 04 (2007) 049 [hep-th/0612157] [INSPIRE].

[42] J. Casalderrey-Solana and D. Teaney, Transverse Momentum Broadening of a Fast Quark in a $N=4$ Yang-Mills Plasma, JHEP 04 (2007) 039 [hep-th/0701123] [INSPIRE].

[43] Y. Hatta, E. Iancu and A.H. Mueller, Jet evolution in the $N=4$ SYM plasma at strong coupling, JHEP 05 (2008) 037 [arXiv:0803.2481] [INSPIRE].

[44] P.M. Chesler, K. Jensen, A. Karch and L.G. Yaffe, Light quark energy loss in strongly-coupled $N=4$ supersymmetric Yang-Mills plasma, Phys. Rev. D 79 (2009) 125015 [arXiv:0810.1985] [INSPIRE].

[45] F. D'Eramo, H. Liu and K. Rajagopal, Transverse Momentum Broadening and the Jet Quenching Parameter, Redux, Phys. Rev. D 84 (2011) 065015 [arXiv:1006.1367] [inSPIRE].

[46] J.M. Maldacena, The Large $N$ limit of superconformal field theories and supergravity, Int. J. Theor. Phys. 38 (1999) 1113 [Adv. Theor. Math. Phys. 2 (1998) 231] [hep-th/9711200] [INSPIRE].

[47] C.W. Bauer, D. Pirjol and I.W. Stewart, Factorization and endpoint singularities in heavy to light decays, Phys. Rev. D 67 (2003) 071502 [hep-ph/0211069] [INSPIRE].

[48] C.W. Bauer, D. Pirjol and I.W. Stewart, On Power suppressed operators and gauge invariance in SCET, Phys. Rev. D 68 (2003) 034021 [hep-ph/0303156] [INSPIRE].

[49] C.W. Bauer, S. Fleming, D. Pirjol and I.W. Stewart, An Effective field theory for collinear and soft gluons: Heavy to light decays, Phys. Rev. D 63 (2001) 114020 [hep-ph/0011336] [INSPIRE].

[50] C.W. Bauer and I.W. Stewart, Invariant operators in collinear effective theory, Phys. Lett. B 516 (2001) 134 [hep-ph/0107001] [INSPIRE].

[51] C.W. Bauer, S. Fleming, D. Pirjol, I.Z. Rothstein and I.W. Stewart, Hard scattering factorization from effective field theory, Phys. Rev. D 66 (2002) 014017 [hep-ph/0202088] [INSPIRE].

[52] T. Becher, A. Broggio and A. Ferroglia, Introduction to Soft-Collinear Effective Theory, Lect. Notes Phys. 896 (2015) 1 [arXiv:1410.1892] [INSPIRE].

[53] G. Ovanesyan and I. Vitev, Medium-induced parton splitting kernels from Soft Collinear Effective Theory with Glauber gluons, Phys. Lett. B 706 (2012) 371 [arXiv:1109.5619] [INSPIRE].

[54] Y.-T. Chien and I. Vitev, Towards the understanding of jet shapes and cross sections in heavy ion collisions using soft-collinear effective theory, JHEP 05 (2016) 023 [arXiv:1509.07257] [INSPIRE].

[55] G. Ovanesyan and I. Vitev, An effective theory for jet propagation in dense QCD matter: jet broadening and medium-induced bremsstrahlung, JHEP 06 (2011) 080 [arXiv:1103.1074] [INSPIRE]. 
[56] Y.-T. Chien, A. Emerman, Z.-B. Kang, G. Ovanesyan and I. Vitev, Jet Quenching from QCD Evolution, Phys. Rev. D 93 (2016) 074030 [arXiv:1509.02936] [InSPIRE].

[57] Z.-B. Kang, R. Lashof-Regas, G. Ovanesyan, P. Saad and I. Vitev, Jet quenching phenomenology from soft-collinear effective theory with Glauber gluons, Phys. Rev. Lett. 114 (2015) 092002 [arXiv:1405.2612] [INSPIRE].

[58] I.Z. Rothstein and I.W. Stewart, An Effective Field Theory for Forward Scattering and Factorization Violation, JHEP 08 (2016) 025 [arXiv: 1601.04695] [INSPIRE].

[59] H.P. Breuer and F. Petruccione, The Theory of Open Quantum Systems, Oxford University Press (2002).

[60] G. Schaller, Open Quantum Systems far from Equilibrium, Lect. Notes Phys. 881 (2014) 1.

[61] C. Young and K. Dusling, Quarkonium above deconfinement as an open quantum system, Phys. Rev. C 87 (2013) 065206 [arXiv: 1001.0935] [InSPIRE].

[62] N. Borghini and C. Gombeaud, Heavy quarkonia in a medium as a quantum dissipative system: Master equation approach, Eur. Phys. J. C 72 (2012) 2000 [arXiv:1109.4271] [INSPIRE].

[63] Y. Akamatsu and A. Rothkopf, Stochastic potential and quantum decoherence of heavy quarkonium in the quark-gluon plasma, Phys. Rev. D 85 (2012) 105011 [arXiv:1110.1203] [INSPIRE].

[64] Y. Akamatsu, Heavy quark master equations in the Lindblad form at high temperatures, Phys. Rev. D 91 (2015) 056002 [arXiv: 1403.5783] [INSPIRE].

[65] J.-P. Blaizot, D. De Boni, P. Faccioli and G. Garberoglio, Heavy quark bound states in a quark-gluon plasma: Dissociation and recombination, Nucl. Phys. A 946 (2016) 49 [arXiv: 1503.03857] [INSPIRE].

[66] R. Katz and P.B. Gossiaux, The Schrödinger-Langevin equation with and without thermal fluctuations, Annals Phys. 368 (2016) 267 [arXiv: 1504.08087] [INSPIRE].

[67] S. Kajimoto, Y. Akamatsu, M. Asakawa and A. Rothkopf, Dynamical dissociation of quarkonia by wave function decoherence, Phys. Rev. D 97 (2018) 014003 [arXiv:1705.03365] [InSPIRE].

[68] D. De Boni, Fate of in-medium heavy quarks via a Lindblad equation, JHEP 08 (2017) 064 [arXiv: 1705.03567] [INSPIRE].

[69] J.-P. Blaizot and M.A. Escobedo, Quantum and classical dynamics of heavy quarks in a quark-gluon plasma, JHEP 06 (2018) 034 [arXiv:1711.10812] [INSPIRE].

[70] J.-P. Blaizot and M.A. Escobedo, Approach to equilibrium of a quarkonium in a quark-gluon plasma, Phys. Rev. D 98 (2018) 074007 [arXiv: 1803.07996] [InSPIRE].

[71] Y. Akamatsu, M. Asakawa, S. Kajimoto and A. Rothkopf, Quantum dissipation of a heavy quark from a nonlinear stochastic Schrödinger equation, JHEP 07 (2018) 029 [arXiv: 1805.00167] [INSPIRE].

[72] T. Miura, Y. Akamatsu, M. Asakawa and A. Rothkopf, Quantum Brownian motion of a heavy quark pair in the quark-gluon plasma, Phys. Rev. D 101 (2020) 034011 [arXiv:1908.06293] [INSPIRE].

[73] N. Brambilla, A. Pineda, J. Soto and A. Vairo, Potential NRQCD: An Effective theory for heavy quarkonium, Nucl. Phys. B 566 (2000) 275 [hep-ph/9907240] [INSPIRE].

[74] N. Brambilla, A. Pineda, J. Soto and A. Vairo, Effective Field Theories for Heavy Quarkonium, Rev. Mod. Phys. 77 (2005) 1423 [hep-ph/0410047] [INSPIRE]. 
[75] S. Fleming and T. Mehen, Doubly heavy baryons, heavy quark-diquark symmetry and NRQCD, Phys. Rev. D 73 (2006) 034502 [hep-ph/0509313] [INSPIRE].

[76] N. Brambilla, M.A. Escobedo, J. Soto and A. Vairo, Quarkonium suppression in heavy-ion collisions: an open quantum system approach, Phys. Rev. D 96 (2017) 034021 [arXiv: 1612.07248] [INSPIRE].

[77] N. Brambilla, M.A. Escobedo, J. Soto and A. Vairo, Heavy quarkonium suppression in a fireball, Phys. Rev. D 97 (2018) 074009 [arXiv:1711.04515] [INSPIRE].

[78] N. Brambilla, M.A. Escobedo, A. Vairo and P. Vander Griend, Transport coefficients from in medium quarkonium dynamics, Phys. Rev. D 100 (2019) 054025 [arXiv:1903.08063] [INSPIRE].

[79] X. Yao and T. Mehen, Quarkonium in-medium transport equation derived from first principles, Phys. Rev. D 99 (2019) 096028 [arXiv: 1811.07027] [InSPIRE].

[80] X. Yao, W. Ke, Y. Xu, S. Bass, T. Mehen and B. Müller, Fate of Heavy Quark Bound States inside Quark-Gluon Plasma, in 18th International Conference on Hadron Spectroscopy and Structure, pp. 688-693 (2020) [DOI] [arXiv:1912.01633] [INSPIRE].

[81] X. Yao, W. Ke, Y. Xu, S.A. Bass, T. Mehen and B. Müller, Quarkonium Production in Heavy Ion Collisions: From Open Quantum System to Transport Equation, Nucl. Phys. A 1005 (2021) 121854 [arXiv:2002.04079] [INSPIRE].

[82] C. Lee, P. Shrivastava and V. Vaidya, Predictions for energy correlators probing substructure of groomed heavy quark jets, JHEP 09 (2019) 045 [arXiv: 1901.09095] [INSPIRE].

[83] Y. Makris and V. Vaidya, Transverse Momentum Spectra at Threshold for Groomed Heavy Quark Jets, JHEP 10 (2018) 019 [arXiv:1807.09805] [INSPIRE].

[84] V. Vaidya and X. Yao, Transverse momentum broadening of a jet in quark-gluon plasma: an open quantum system EFT, JHEP 10 (2020) 024 [arXiv: 2004.11403] [INSPIRE].

[85] A.J. Larkoski, S. Marzani, G. Soyez and J. Thaler, Soft Drop, JHEP 05 (2014) 146 [arXiv: 1402.2657] [INSPIRE].

[86] A.J. Larkoski, I. Moult and D. Neill, Power Counting to Better Jet Observables, JHEP 12 (2014) 009 [arXiv: 1409.6298] [INSPIRE].

[87] A.J. Larkoski, I. Moult and B. Nachman, Jet Substructure at the Large Hadron Collider: A Review of Recent Advances in Theory and Machine Learning, Phys. Rept. 841 (2020) 1 [arXiv: 1709.04464] [INSPIRE].

[88] D. Gutierrez-Reyes, Y. Makris, V. Vaidya, I. Scimemi and L. Zoppi, Probing Transverse-Momentum Distributions With Groomed Jets, JHEP 08 (2019) 161 [arXiv: 1907.05896] [INSPIRE].

[89] A.V. Manohar, T. Mehen, D. Pirjol and I.W. Stewart, Reparameterization invariance for collinear operators, Phys. Lett. B 539 (2002) 59 [hep-ph/0204229] [INSPIRE].

[90] J.-Y. Chiu, A. Jain, D. Neill and I.Z. Rothstein, A Formalism for the Systematic Treatment of Rapidity Logarithms in Quantum Field Theory, JHEP 05 (2012) 084 [arXiv:1202.0814] [INSPIRE].

[91] V. Vaidya, Probing a dilute short lived Quark Gluon Plasma medium with jets, in preparation.

[92] V. Vaidya, Radiative corrections for factorized jet observables in heavy ion collisions, arXiv: 2107.00029 [INSPIRE]. 
[93] I.W. Stewart, F.J. Tackmann and W.J. Waalewijn, Factorization at the LHC: From PDFs to Initial State Jets, Phys. Rev. D 81 (2010) 094035 [arXiv:0910.0467] [INSPIRE].

[94] A. Hornig, Y. Makris and T. Mehen, Jet Shapes in Dijet Events at the LHC in SCET, JHEP 04 (2016) 097 [arXiv: 1601.01319] [INSPIRE].

[95] C.W. Bauer, F.J. Tackmann, J.R. Walsh and S. Zuberi, Factorization and Resummation for Dijet Invariant Mass Spectra, Phys. Rev. D 85 (2012) 074006 [arXiv:1106.6047] [InSPIRE].

[96] S.D. Ellis, C.K. Vermilion, J.R. Walsh, A. Hornig and C. Lee, Jet Shapes and Jet Algorithms in SCET, JHEP 11 (2010) 101 [arXiv:1001.0014] [INSPIRE].

[97] M.G. Echevarria, I. Scimemi and A. Vladimirov, Universal transverse momentum dependent soft function at NNLO, Phys. Rev. D 93 (2016) 054004 [arXiv:1511.05590] [INSPIRE]. 